\title{
Genus-group names of Afrotropical flea beetles (Coleoptera: Chrysomelidae: Alticinae): Annotated catalogue and biogeographical notes
}

\author{
Maurizio BIONDI and Paola D’ALESSANDRO
}

\author{
Dipartimento di Scienze Ambientali, University of L'Aquila, 67100 Coppito-L'Aquila, Italy; \\ e-mails: maurizio.biondi@univaq.it; paola.dalessandro@univaq.it
}

Key words. Taxonomy, Afrotropical region, Chrysomelidae, Alticinae, Galerucinae, flea beetle genera, catalogue, synonymies, new combinations.

\begin{abstract}
This paper consists of an up to date annotated catalogue of the Afrotropical genera of Alticinae (Chrysomelidae), with biogeographical notes on the flea beetle fauna occurring in Sub-Saharan Africa and Madagascar. The following new synonymies are proposed: Eugonotes Jacoby, 1897 (a subgenus of Sanckia Duvivier, 1891) = Brancucciella Medvedev, 1995 syn. n.; Amphimela Chapuis, 1875 = Dibolosoma Jacoby, 1897 syn. n.; Amphimela Chapuis, 1875 = Halticova Fairmaire, 1898 syn. n.; Podagrica Chevrolat, 1837 = Podagrixena Bechyné, 1968 syn. n.; Aphthona Chevrolat, 1837 = Pseudeugonotes Jacoby, 1899 syn. n.; Nisotra Baly, 1864 = Pseudonisotra Bechyné, 1968 syn. n. The following new combinations are proposed: Afrorestia sjostedti (Weise, 1910) comb. n. (from Crepidodera); Bechuana natalensis (Jacoby, 1906) comb. n. (from Ochrosis); Sesquiphaera natalensis (Jacoby, 1906) comb. n. (from Sphaeroderma). The genus Hildenbrandtina Weise, 1910 is trasferred from Galerucinae to Alticinae. New distributional data for many genera in the Afrotropical region is provided.
\end{abstract}

\section{INTRODUCTION}

The Chrysomelidae is one of the largest phytophagous insect families and includes approximately 37,000 to 40,000 species (Jolivet \& Verma, 2002). The relationship between two of its subfamilies, the monophyletic Alticinae (commonly termed flea beetles) and closely related Galerucinae is an area of active research on Chrysomelidae phylogeny (Duckett et al., 2004; Gómez-Zurita et al., 2007). In this paper, the Alticinae and Galerucinae are considered to be separate subfamilies because of the metafemoral spring in Alticinae and specific structures of the spermatheca, median lobe of aedeagus and hind wing venation (cf. Furth \& Suzuki, 1994, 1998). The Alticinae includes 4,000 to 8,000 species grouped in approximately 500 genera that are distributed worldwide, although most occur in the tropical regions of South America, Africa and Asia (Konstantinov \& Vandenberg, 1996; Santiago-Blay, 2004). They are highly specialized phytophagous insects, of which the adults and larvae feed on stems, leaves and roots of most of the higher plant families (Konstantinov \& Vandenberg, 1996).

Details of the composition of the Afrotropical flea beetle fauna are incomplete, which is highlighted by the discrepancy in the numbers of morphogenera and morphospecies in African entomological material preserved in public and private collections and those that have been officially described. Current scientific literature includes approximately 300 research papers dedicated in whole or part to Sub-Saharan and Madagascan Chrysomelidae Alticinae, including publications on taxonomy, faunistics and ecology. The chronological trend in the numbers of these publications in time is summarized in Fig. 1, begin- ning as early as 1830 , although the first significant contribution on the Afrotropical (including Madagascar) flea beetle fauna was by the English coleopterist, Joseph Sugar Baly (1816-1890). Subsequently, in the twenty years following Baly's death (1890-1910) there were three important entomologists working on these beetles: Léon Fairmaire (1820-1906), a French specialist on Coleoptera and Hemiptera; Julius Weise (1884-1925), a German coleopterist that, despite his short life, published a large number of scientific papers, not only on Chrysomelidae but also on Coccinellidae, Curculionoidea, etc.; and Martin Jacoby (1842-1907), a German musician and coleopterist who published 150 articles on leaf beetles after moving to London.

A decrease in the number of publications on Afrotropical flea beetle fauna followed, until the revival in 1930-1940 initiated by the English coleopterist Gilbert Ernest Bryant (1878-1965) and the French chrysomelid specialist Victor Laboissière (1875-1942). The Alticinae specialists Jan Bechyné (1920-1973) and Gerhard Scherer then published many monographs (see References) on the flea beetle fauna of Sub-Saharan Africa and, to a less extent, Madagascar, describing many new genera and species from 1950-1970. More recently, contributions on the Afrotropical flea beetle fauna were published by Gerhard Scherer, Maurizio Biondi, Paola D’Alessandro, Manfred Döberl and Serge Doguet (see References).

\section{MATERIAL AND METHODS}

The catalogue is arranged alphabetically by genus. Names that are in square brackets refer to synonymies, genera incorrectly reported in the Afrotropical region or, in some cases, to 


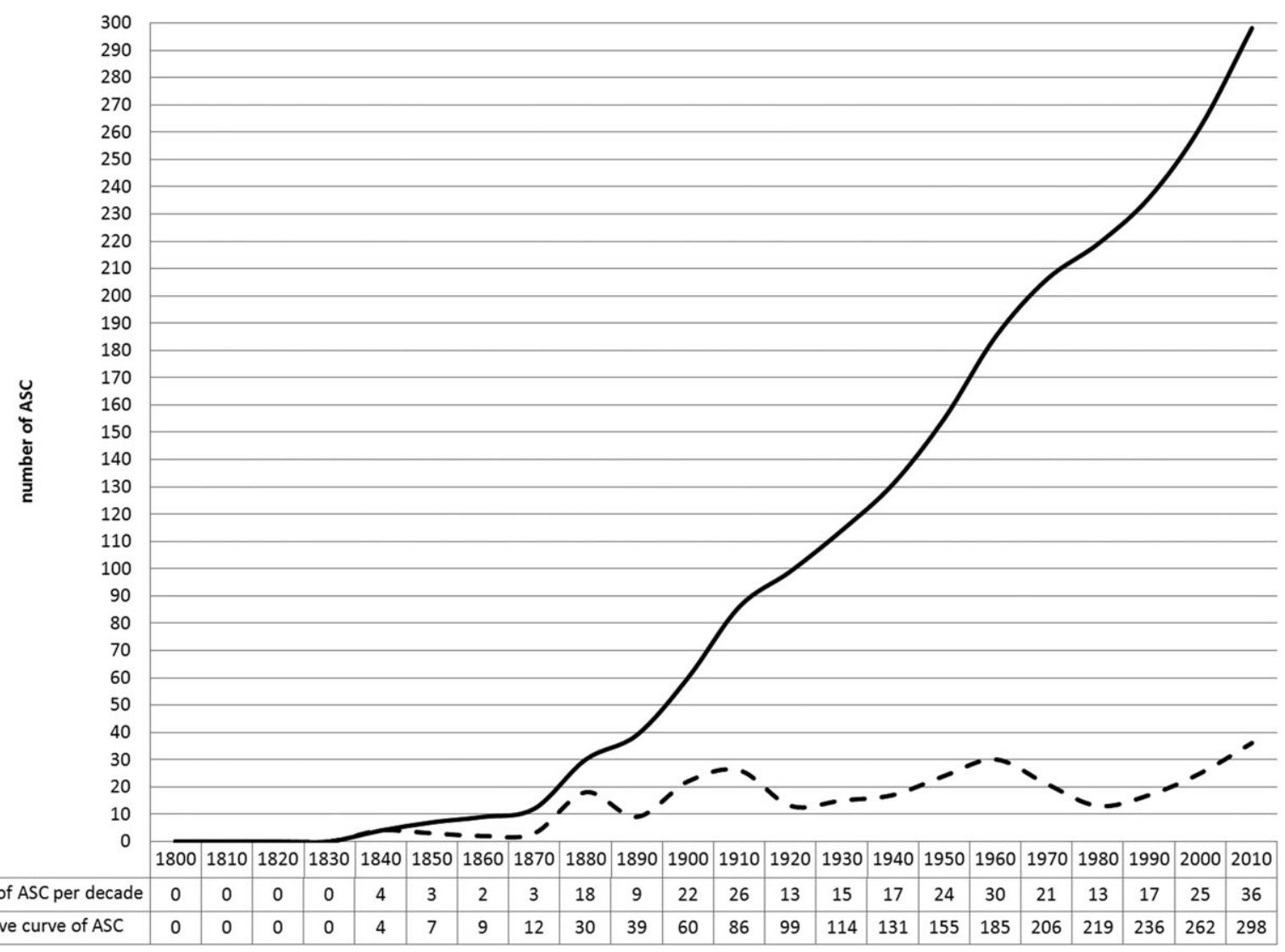

Fig. 1. Chronology of the publications on the Afrotropical flea beetle fauna (ASC).

genera transferred to Galerucinae or to genus-group names that are unavailable.

In addition to author and date of publication, each genusgroup name is accompanied by: (a) synonymies, exclusively those for the Afrotropical region; (b) bibliographic references, including the original description and other important taxonomical contributions; (c) type species, including the method of species assignment; (d) geographic distribution in the Afrotropical region (cf. Graf \& Cummings, 2007) and other zoogeographical regions (cf. Sclater, 1858); (e) ecological remarks, mainly host-plants and/or habitat preferences; (f) notes, including the number of Afrotropical species and important taxonomical news.

The type material examined for this study is preserved in the following institutions: BAQ - collection of the author, Dipartimento di Scienze Ambientali, University of L'Aquila, Italy; BMNH - The Natural History Museum, London, United Kingdom; ISNB - Institut Royal des Sciences Naturelles de Belgique, Brussels, Belgium; MNHN - Muséum National d'Histoire Naturelle, Paris, France; MCSN - Museo Civico di Storia Naturale "Giacomo Doria", Genova, Italy; MRAC Musée Royal de l'Afrique Centrale, Tervuren, Belgium; MZLU - Lund University, Sweden; NHMB - Naturhistorisches Museum, Basel, Switzerland; NHRS - Naturhistoriska Riksmuseet, Stockholm, Sweden; SANC - South African National Collection, ARC-Plant Protection Research Institute, Pretoria, South Africa; SMNS - Staatliches Museum für Naturkunde, Stuttgart, Germany; TMSA - Transvaal Museum, Pretoria, South Africa; ZMHB - Museum für Naturkunde der Humboldt-Universität, Berlin, Germany; ZSMC - Zoologische Staatssammlung, Munich, Germany.

\section{Abbreviations}

Regions: AFR - Afrotropical; AUS - Australian; CAF - Central Afrotropical; EAF - Eastern Afrotropical; IND - IndoMalayan; NEA - Nearctic; NEO - Neotropical; PAL Palaearctic; SAF - Southern Afrotropical; WAF - Western Afrotropical. MAD - Madagascar; MAS - Mascarene Islands; SEY - Seychelles Islands; SSA - Sub-Saharan Africa; (?) record to be confirmed; (!) - new record.

\section{CATALOGUE OF AFROTROPICAL FLEA BEETLE GENERA}

\section{Abrarius Fairmaire, 1902}

=Entymosina Weise, 1910 (synonymized by Bechyné, 1958c)

References. Fairmaire, 1902: 261; Weise, 1910b: 438; Bechyné, 1947a: 44 (sub Entymosina); 1958c: 9.

Type species. Abrarius cribrosus Fairmaire, 1902: 261 (Madagascar: Plateau de l'Ankara), by monotypy.

Distribution. Madagascar.

Ecology. No information.

Notes. Genus endemic to Madagascar and consisting of about ten species. Abrarius is very similar to the Neotropical genus Gioia Bechyné (1955e: 77), which could be a synonym.

\section{Afroaltica Biondi \& D'Alessandro, 2007}

References. Biondi \& D'Alessandro, 2007: 99: D'Alessandro \& Biondi, in press.

Type species. Afroaltica subaptera Biondi \& D'Alessandro, 2007: 100 (Republic of South Africa, KwaZulu-Natal, Karkloof area), by original designation.

Distribution. Republic of South Africa (Limpopo, Mpumalanga and KwaZulu-Natal). 
Ecology. A. subaptera collected in an open field on Graminae (Biondi \& D'Alessandro, 2007).

Notes. Two species.

\section{[Afroalytus Scherer, 1961]}

=Manobia Jacoby, 1885

\section{Afrocrepis Bechyné, 1954}

References. Bechyné, 1954b: 680; Heikertinger, 1925: 99 (sub Derocrepis Weise, 1886).

Type species. Crepidodera carinipennis Jacoby, 1903a: 12 (KwaZulu-Natal, Malvern), by original designation.

Distribution. Republic of South Africa.

Ecology. No information.

Notes. Three species. Crepidodera betiokyensis Bechyné (1954a: 46), erroneously attributed by Bechyné (1964: 152) to this genus, must be placed in Afrorestia Bechyné (cf. Scherer, 1962b: 57; pers. data).

\section{Afrorestia Bechyné, 1959}

References. Bechyné, 1959b: 232.

Type species. Crepidodera laeviuscula Csiki, 1940: 297 (West Africa), by original designation.

Distribution. Ethiopia, Democratic Republic of Congo, Uganda, Rwanda, Burundi, Tanzania, Zimbabwe, Republic of South Africa and Madagascar.

Ecology. Some species of this genus were collected on Apiaceae plants in South Africa (pers. data).

Notes. About twenty species. Crepidodera betiokyensis Bechyné (1954a: 46), erroneously attributed by Bechyné (1964: 152) to Afrocrepis Bechyné, must be placed in this genus (cf. Scherer, 1962b: 57; pers. data). Crepidodera sjostedti Weise (1910a: 221) from Kilimanjaro was incorrectly attributed to Asiorestia Jacobson, 1925 (a genus not occurring in the Afrotropical region) by Bechyné (1957). This species is here transferred to Afrorestia Bechyné as Afrorestia sjostedti (Weise, 1910) comb. $\mathrm{n}$.

\section{[Allomorpha Jacoby, 1892]}

=Hespera Weise, 1889

\section{Alocypha Weise, 1911}

References. Weise, 1911: 170.

Type species. Alocypha litura Weise, 1911: 171 (East Africa: Lindi) [=Aphthona bimaculata Jacoby, 1903a: 11 (KwaZuluNatal)], by monotypy.

Distribution. Tanzania, Zambia(!), Malawi, Botswana(!), Mozambique(!), Namibia(!) and Republic of South Africa (KwaZulu-Natal).

Ecology. Alocypha bimaculata is a harmful pest of Sesame (Sesamum indicum L.) crops, especially in Tanzania (Mponda et al., 1997).

Notes. One species.

\section{Altica Geoffroy, 1762}

=Haltica Illiger, 1801 (unjustified emendation)

$=$ Graptodera Chevrolat, 1837 (synonymized by Weise, 1888)

References. Geoffroy, 1762: 244; Illiger, 1801: 138; Chevrolat, 1837: 388; Weise, 1888: 825; Allard, 1889: 43 (sub Graptodera); Bechyné, 1954a: 43; 1955a: 209; 1960a: 77; Döberl, 2008: 35; 2010: 51.

Type species. Chrysomela oleracea Linnaeus, 1758: 372 (Europe), by subsequent designation by Latreille (1810: 432).

Distribution. All zoogeographical regions.
Ecology. Polyphagous genus associated with herbaceous plants, shrubs and trees belonging to several plant families (cf. Jolivet \& Hawkeswood, 1995).

Notes. Thirty-five species in Sub-Saharan Africa and 8 in Madagascar.

\section{Amphimela Chapuis, 1875}

=Diboloides Jacoby, 1897 (synonymized by Scherer, 1961)

=Dibolosoma Jacoby, 1897 syn. n.

=Halticova Fairmaire, 1898 syn. n.

=Halticella Jacoby, 1899 (nec Halticella Stephens, 1829: 36, Hymenoptera, Chalcidoidea).

$=$ Cercyonia Weise, 1901 (synonymized by Scherer, 1961)

=Halticorthaea Csiki, 1940 (new name for Halticella Jacoby, 1899; synonymized by Scherer, 1961)

References. Chapuis, 1875: 34; Baly, 1875: 27; Jacoby, 1897: 553, 559; Fairmaire, 1898: 428; Jacoby, 1899b: 357; Weise, 1901: 303; Maulik, 1929: 207 (sub Diboloides); Csiki in Heikertinger \& Csiki, 1940: 418; Scherer, 1961: 252.

Type species. Amphimela mouhoti Chapuis, 1875: 36 (Indonesia), by monotypy.

Distribution. Afrotropical (including Madagascar), IndoMalayan and Australian regions.

Ecology. Amphimela bryanti (Csiki in Heikertinger \& Csiki, 1940) on Bersamia sp. (Melianthaceae) in Uganda (Bryant, 1936). A. citri (Bryant, 1922) cited as a harmful pest of citrus in western Africa (Bryant, 1922 : 474).

Notes. About thirty species in the Afrotropical region. Bechyné (1964: 161) established the synonymy between Dibolosoma quadripustulatum Jacoby, 1897 [sub 4-punctata (sic!)] and Halticova rufoguttata Fairmaire, 1898.

\section{Anaxerta Fairmaire, 1902}

References. Fairmaire, 1902: 267.

Type species. Anaxerta castanea Fairmaire, 1902: 268 (Madagascar: Ankarahitra), by monotypy.

Distribution. Madagascar.

Ecology. No information.

Notes. One species.

\section{Angulaphthona Bechyné, 1960}

References. Bechyné, 1960a: 74; Scherer, 1978: 265; Medvedev, 1996: 261.

Type species. Aphthona heteromorpha Bechyné, 1955c: 62 (Madagascar: Bas Mangoky), by original designation.

Distribution. Egypt, Tchad, Sudan, Somaliland, Sierra Leone(!), Nigeria, Democratic Republic of Congo, Uganda(!), Mozambique, Madagascar and Arabian Peninsula (Saudi Arabia and North Yemen).

Ecology. Angulaphthona heteromorpha collected on cotton plants, Gossypium sp. (Malvaceae) (Bechyné 1955c, sub Aphthona).

Notes. Five species in the Afrotropical region.

\section{Antanemora Bechyné, 1964}

Lactica Erichson, 1847 (pars)

References. Erichson, 1847: 173; Bechyné, 1950: 220 (sub Lactica); 1964: 145; Bechyné \& Bechyné, 1975: 26.

Type species. Lactica carbonaria Bechyné, 1948a: 7 (Madagascar: Environs de Rogez; Ankazoabo), by original designation.

Distribution. Madagascar.

Ecology. No information.

Notes. About twenty species. 


\section{Aphthona Chevrolat, 1837}

=Pseudeugonotes Jacoby, 1899 syn. $\mathrm{n}$.

References. Chevrolat, 1837: 415; Jacoby, 1899a: 531; Bechyné, 1960a: 67.

Type species. Altica cyparissiae Koch, 1803: 80 (Europe), by subsequent designation by Chûjô (1937: 119).

Distribution. Afrotropical (including Madagascar), Palaearctic and Indo-Malayan regions. All the species from the Neotropical and Nearctic regions described as Aphthona should be attributed to different genera (cf. Konstantinov \& Vanderberg, 1996; Konstantinov, 1998).

Ecology. Genus mainly associated with Euphorbiaceae, but also with Geraniaceae, Cistaceae, Rosaceae, Linaceae, Iridaceae and Lythraceae (cf. Jolivet \& Hawkeswood, 1995).

Notes. About one hundred species in Sub-Saharan Africa and Madagascar. The examination of the holotype of Pseudeugonotes vannutellii Jacoby (1899a: 531) (Somaliland) resulted in the following new synonymy: Aphthona Chevrolat, $1837=$ Pseudeugonotes Jacoby, 1899 syn. n.

\section{Argopistes Motschulsky, 1860}

References. Motschulsky, 1860: 236; Weise, 1895: 335; Bryant, 1922a: 474.

Type species. Argopistes biplagiata Motschulsky, 1860: 236 (Siberia), by monotypy.

Distribution. Central, eastern and southern Africa, and Madagascar; eastern Palaearctic, Nearctic, northern Neotropical, Indo-Malayan and Australian regions.

Ecology. Many species of this genus are associated with Oleaceae in Sub-Saharan Africa, especially with Olive trees [Olea europaea var. africana (Mill.)], on which the larvae are leaf miners and adults defoliators (cf. Jolivet \& Hawkeswood, 1995; pers. data).

Notes. About fifteen species in Sub-Saharan Africa and Madagascar (pers. data)

\section{[Argopus Fischer, 1824]}

Not present in the Afrotropical region.

References. Fischer, 1824: 184; Weise, 1902a: 171; Laboissière, 1941: 319.

Notes. Argopus maculiceps Boheman (1859: 200) was transferred by Laboissière (1941) to the genus Toxaria Weise; $A$. pusillus Gerstaecker (1871: 85) was transferred by Weise (1902a) to the genus Sphaeroderma Stephens.

\section{[Argosomus Wollaston, 1867]}

=Sphaeroderma Stephens, 1831 .

[Aridohespera Selman, 1963]

=Eriotica Harold, 1877

\section{[Asiorestia Jacobson, 1925]}

Not present in the Afrotropical region.

References. Jacobson, 1925: 274; Bechyné, 1957: 181; 1959b: 233.

Notes. Bechyné (1957) attributed Crepidodera sjostedti Weise (1910a: 221) from Kilimanjaro to this genus. This species is here transferred to Afrorestia Bechyné as Afrorestia sjostedti (Weise, 1910) comb. n.

\section{[Asphaera Chevrolat, 1843]}

Not present in the Afrotropical region.

References. Chevrolat, 1843: 227; Fairmaire, 1886: 93; Jacoby, 1892: 573; Bechyné, 1959c: 319.
Notes. The three species wrongly attributed to this Neotropical genus were transferred by Bechyné (1959c) to the genera Physomandroya Bechyné and Hemipyxis Chevrolat.

\section{Bangalaltica Bechyné, 1960}

References. Bechyné, 1960b: 9; Biondi \& D’Alessandro, 2003: 104.

Type species. Bangalaltica antennalis Bechyné, 1960b: 9 (Belgian Congo: Bangala; Lubutu-Kituri), by monotypy.

Distribution. Congo(!) and Democratic Republic of Congo.

Ecology. No information.

Notes. One species.

\section{[Balanomorpha Chevrolat, 1837]}

=Mantura Stephens, 1831

\section{Bechuana Scherer, 1970}

References. Scherer, 1970: 301.

Type species. Bechuana nigripes Scherer, 1970: 302 (Free State: Boshof; North-West Province: Vryburg), by original designation.

Distribution. Republic of South Africa [North-West Province(!), Gauteng, KwaZulu-Natal, Free State, Western and Eastern Provinces].

Ecology. No information.

Notes. Two species. Ochrosis natalensis Jacoby (1906: 17) is here attributed to this genus as Bechuana natalensis (Jacoby, 1906) comb. n.

\section{Bechynella Biondi \& D’Alessandro, 2010}

Serraphula Jacoby, 1897 (pars)

References. Biondi \& D’Alessandro, 2010: 28; Bechyné, 1955b: 517 (sub Serraphula); 1959a: 13 (sub Serraphula).

Type species. Serraphula bohumilae Bechyné, 1955b: 517 (French Guinea: Dalaba), by original designation.

Distribution. Guinea, Ivory Coast, Nigeria, Cameroon and Democratic Republic of Congo.

Ecology. No information.

Notes. The three species attributed to this genus were described by Bechyné (1955b, 1959a) under Serraphula Jacoby (cf. Biondi \& D'Alessandro, 2010).

\section{Bikasha Maulik, 1931}

References. Maulik, 1931: 256.

Type species. Bikasha tenuipunctata Maulik, 1931: 257 (Seychelles), by original designation.

Distribution. Indian Ocean (Seychelles). The recent synonymies established by Konstantinov \& Prathapan (2008: 387) indicate that this flea beetle genus occurs also in the IndoMalayan region.

Ecology. In Seychelles, B. tenuipunctata Maulik and B. fortipunctata Maulik (1931: 258) were collected in forest, and $B$. minor Maulik (1931: 259) in coastal wet meadows.

Notes. Three species in Seychelles.

\section{Biodontocnema Biondi, 2000}

References. Biondi, 2000: 347; 2002b: 356.

Type species. Biodontocnema brunnea Biondi, 2000: 348 (Namibia, Kaross), by monotypy.

Distribution. Namibia.

Ecology. The only species in this genus is associated with moist habitats (Biondi, 2000).

Notes. One species.

Blepharida Chevrolat, 1837

includes subgen. Blepharidina Bechyné, 1968 
=Eutheca Baly, 1878 (nec Eutheca Kiesenwetter in Erichson, 1877: 155, Coleoptera: Anobiidae).

=Calotheca Heyden, 1887 (new name for Eutheca Baly, 1878; synonymized by Scherer, 1961)

=Blepharidella Weise, 1910 (synonymized by Scherer, 1961)

=Blepharidula Weise, 1916 (an unnecessary new name for Eutheca Baly, 1878).

References. Chevrolat, 1837: 418; Heyden, 1887: 98; Duvivier, 1891: 242; Weise, 1910a: 220; Laboissière, 1942: 95; Bryant, 1944a: 129; Scherer, 1961: 252; Bechyné, 1968: 1725; Furth, 1998: 12; Furth \& Lee, 2000: 26; Becerra, 2004: 116.

Type species. Chrysomela rhois Forster, 1771: 21 (North America), by subsequent designation by Chevrolat (1842: 606).

Distribution. Afrotropical (including Madagascar), southern Palaearctic (Israel), Nearctic and Neotropical regions.

Ecology. The Afrotropical species of Blepharida are generally associated with shrubs of Rhus (Anacardiaceae) (Furth \& Young, 1988: 496; pers. data).

Notes. About thirty-five species in Sub-Saharan Africa and two in Madagascar. Mainly the nominotypical subgenus Blepharida is widespread in Nearctic and Neotropical regions and the subgenus Blepharidina in the Afrotropical region (Furth, 1998).

\section{Blepharidina Bechyné, 1968}

subgenus of Blepharida Chevrolat, 1837

References. Bechyné, 1968: 1725; Furth, 1998: 12.

Type species. Blepharida guttulata Baly, 1881: 52 (Angola), by original designation.

Distribution. Afrotropical (including Madagascar) and southern Palaearctic (Israel) regions.

Ecology. Genus generally associated with shrubs of Rhus (Anacardiaceae) (Furth \& Young, 1988: 496; pers. data).

Notes. About thirty-five species in Sub-Saharan Africa and two in Madagascar.

\section{[Blepharidula Weise, 1916]}

$=$ Blepharida Chevrolat, 1837

References. Weise, 1916: 39; Baly, 1878: 204; Heyden, 1887: 98.

Notes. An unnecessary new name proposed by Weise (1916) for Eutheca Baly, 1878 (nec Eutheca Kiesenwetter in Erichson, 1877: 155, Coleoptera: Anobiidae). Weise incorrectly regarded the previous replacement name for Eutheca (Calotheca Heyden, 1887) as unavailable because it was already in use for a plant genus of Graminae (cf. Palisot de Beauvois, 1812: 85).

[Brinckaltica Bechyné, 1959]

=Chaetocnema Stephens, 1831

\section{Buphonella Jacoby, 1903}

References. Jacoby, 1903a: 37; Laboissière, 1922: 178.

Type species. Apophylia murina Gerstaecker, 1871: 83 (Zanzibar), by subsequent designation by Laboissière (1922: 179).

Distribution. Central and eastern Africa.

Ecology. Genus associated with Graminae. Buphonella murina (Gerstaecker) and B. metallica Jacoby (1907: 523) can damage maize (cf. Drinkwater, 1989: 315).

Notes. Four species.

[Calotheca Heyden, 1887]

=Blepharida Chevrolat, 1837

References. Heyden, 1887: 98; Baly, 1878: 204.
Notes. New name for Eutheca Baly, 1878 preoccupied by Eutheca Kiesenwetter in Erichson, 1877: 155 (Coleoptera, Anobiidae).

\section{Carcharodis Weise, 1910}

References. Weise, 1910b: 434; Bechyné, 1954b: 683; Biondi, 2002b: 356.

Type species. Chaetocnema rugiceps Baly, 1877b: 308 (Madagascar), by subsequent designation by Bechyné (1954b: 683).

Distribution. Central and southern Africa, and Madagascar.

Ecology. Species of this genus live in moist habitats and are probably associated with plants of the family Cyperaceae (pers. data).

Notes. Seven species.

\section{Celisaltica Biondi, 2001}

References. Biondi, 2001: 644.

Type species. Celisaltica ruwenzorica Biondi, 2001: 644 (Ruwenzori), by original designation.

Distribution. Uganda.

Ecology. The only species in this genus lives at high altitudes (3,200-4,000 m a.s.1.) on the Ruwenzori Massif, and is associated with the Ericetum plant community (Biondi, 2001).

Notes. One species.

[Cercyonia Weise, 1901]

=Amphimela Chapuis, 1875

\section{Chaetocnema Stephens, 1831}

=Plectroscelis Chevrolat, 1837 (synonymized by Weise, 1886)

=Exorhina Weise, 1886 (synonymized by Heikertinger \& Csiki, 1939-1940)

=Brinckaltica Bechyné 1959 (synonymized by Scherer, 1961)

References. Stephens, 1831: 325; Chevrolat, 1837: 417; Baly, 1877a: 166; Weise, 1886: 750; Bryant, 1928: 393; Heikertinger \& Csiki, 1940: 376; Laboissière, 1942: 81; Bechyné, 1959b: 236; 1960a: 91; Scherer, 1961: 259; Biondi, 2001b: 233; 2002a: 266; 2002b: 356; Biondi \& D'Alessandro, 2006: 720.

Type species. Chrysomela concinna Marsham, 1802 (Europe), by subsequent designation by Westwood (1840: 42).

Distribution. All zoogeographical regions.

Ecology. Genus mainly associated with Cyperaceae, Juncaceae, Graminae, Polygonaceae and Chenopodiaceae (cf. Jolivet \& Hawkeswood, 1995). In the Afrotropical region some Chaetocnema are serious pests of rice (Biondi \& D'Alessandro, 2008a).

Notes. Over one hundred species in Sub-Saharan Africa and Madagascar.

\section{Chaillucola Bechyné, 1968}

References. Bechyné, 1968: 1713; Biondi \& D’Alessandro, 2003: 104.

Type species. Chaillucola formicicornis Bechyné, 1968: 1714 (Congo: Mbila), by original designation.

Distribution. Congo.

Ecology. No information.

Notes. One species.

\section{[Chaloenus Westwood, 1862]}

Not present in the Afrotropical region.

References. Westwood, 1861: 216; Bryant, 1927: 615; Bechyné, 1955b: 543.

Notes. Chaloenus viridis Bryant, 1927 (=Terpnochlorus perrieri Fairmaire, 1904) was wrongly attributed to this Indo-Malayan genus (Bechyné, 1955b). 


\section{Chirodica Germar, 1834}

References. Germar, 1834: 2; Baly, 1876a: 441; Scherer, 1983: 173; Biondi, 1998b: 17, 45.

Type species. Chirodica chalcoptera Germar, 1834: 16 (Cape of Good Hope), by monotypy.

Distribution. Namibia and Republic of South Africa.

Ecology. Genus strictly associated with the plant family Proteaceae (mainly Protea spp. and Leucadendron spp.) (Biondi, 1998b).

Notes. Eight species.

[Cladocera Hope, 1840]

=Polyclada Chevrolat, 1835

[Cladotelia Kolbe, 1894]

=Polyclada Chevrolat, 1835

\section{Collartaltica Bechyné, 1959}

References. Bechyné, 1959a: 27; Biondi, 2002b: 358; Biondi \& D'Alessandro, 2004: 286.

Type species. Collartaltica cryptostoma Bechyné, 1959a: 27 (Belgian Congo: Faradje, Tomati), by monotypy.

Distribution. Nigeria, southern Sudan, Central African Republic, Democratic Republic of Congo, Kenya, Tanzania, Zimbabwe and Republic of South Africa.

Ecology. The genus is associated with Graminae in moist meadows and forest clearings (Biondi \& D'Alessandro, 2004).

Notes. Six species.

\section{[Crepidodera Chevrolat, 1837]}

Not present in the Afrotropical region.

References. Chevrolat, 1837: 415.

Notes. Afrotropical species initially attributed to this genus (cf. Bryant, 1927; Jacoby, 1899b, 1903a, 1906, Weise, 1902b, 1910a, 1924) were mainly transferred to the genera Afrorestia Bechyné, Afrocrepis Bechyné and Orthocrepis Weise.

\section{[Cyrtoma Clark, 1863]}

Nomen nudum.

Notes. Clark (1863: 165) uses this genus-name, without any description, for a never published species from Madagascar ["apicale, Clark" synonymized with "4-maculata, Chevr." and "Madecassae, Chevr." (also the latter two were never published)].

\section{Decaria Weise, 1895}

=Embolimus Weise, 1902

References. Weise, 1895: 344; 1902b: 303; Biondi \& D'Alessandro, 2003: 104.

Type species. Decaria tricolor Weise, 1895: 344 (Sierra Leone, Bang-Haas), by monotypy.

Distribution. Afrotropical region (excluding Madagascar).

Ecology. Genus mainly associated with Boraginaceae (Heliotropium), Sterculiaceae (Cola) and Lamiaceae (Ocimum) (cf. Jolivet \& Hawkeswood, 1995).

Notes. About twenty species.

[Decarthrocera Laboissière, 1937]

Genus transferred to the subfamily Galerucinae (Furth \& Suzuki, 1994).

References. Laboissière, 1937: 27; Furth \& Suzuki, 1994: 131.

[Derocrepis Weise, 1886]

Not present in the Afrotropical region.
References. Weise, 1886: 676, 686; Heikertinger, 1925: 95.

Notes. The species initially attributed to this Palaearctic genus were transferred to the genera Afrocrepis Bechyné and Afrorestia Bechyné.

\section{Diamphidia Gerstaecker, 1855}

References. Gerstaecker, 1855: 638; Baly, 1861: 198; Laboissière, 1942: 109.

Type species. Diamphidia femoralis Gerstaecker, 1855: 638 (Sena and Port Natal), by monotypy.

Distribution. Central, eastern and southeastern Africa.

Ecology. Genus associated with shrubs and trees of Commiphora (Burseraceae) (cf. Jolivet \& Hawkeswood, 1995; Chaboo et al., 2007).

Notes. Seventeen species.

Dibolia Latreille, 1829

=Haltitarsus Berthold, 1827

References. Latreille, 1829: 155; Berthold, 1827: 401; Baly, 1876b: 598; Weise, 1926: 24 (sub Haltitarsus); Bechyné, 1960a: 101.

Type species. Haltica occultans Koch, 1803: 22 (Europe), by subsequent designation by Chûjô (1936: 84).

Distribution. Sub-Saharan Africa (absent in Madagascar); Palaearctic, Nearctic and Neotropical regions.

Ecology. Genus mainly associated with Lamiaceae (cf. Jolivet \& Hawkeswood, 1995).

Notes. About twenty species in Sub-Saharan Africa.

[Diboloides Jacoby, 1897]

=Amphimela Chapuis, 1875

[Dibolosoma Jacoby, 1897]

=Amphimela Chapuis, 1875

\section{Dimonikaea Bechyné, 1968}

References. Bechyné, 1968: 1711; Biondi \& D’Alessandro, 2003: 104.

Type species. Dimonikaea descarpentriesi Bechyné, 1968: 1712 (Congo: Dimonika), by original designation.

Distribution. Congo.

Ecology. No information.

Notes. One species. Bechyné (1968) wrongly included Gabonia miraculosa Scherer (1963: 652) in this genus (pers. data).

Diphaulacosoma Jacoby, 1892

References. Jacoby, 1892a: 574.

Type species. Diphaulacosoma laevipenne Jacoby, 1892: 574-575 (Madagascar), by monotypy.

Distribution. Madagascar.

Ecology. No information.

Notes. One species. Neoderina Bechyné, 1952 (currently placed as a subgenus of Neodera Duvivier, 1891) could be a synonym of Diphaulacosoma (pers. data).

\section{Djallonia Bechyné, 1955}

References. Bechyné, 1955b: 534; Scherer, 1962a: 57.

Type species. Djallonia maindra Bechyné, 1955b: 534 (French Guinea: Dalaba), by monotypy.

Distribution. Guinea and Democratic Republic of Congo.

Ecology. No information.

Notes. One species.

Drakensbergianella Biondi \& D'Alessandro, 2003

References. Biondi \& D'Alessandro, 2003: 100. 
Type species. Drakensbergianella rudebecki Biondi \& D’Alessandro, 2003: 100 (Republic of South Africa, Drakensberg), by monotypy.

Distribution. Republic of South Africa (Free State and KwaZulu-Natal).

Ecology. The only species in this genus lives in alpine meadows (over 2,000 $\mathrm{m}$ a.s.1.) on the Drakensberg and was collected on inflorescences of Asteraceae, such as Senecio and Helichrysum (Biondi \& D'Alessandro, 2003).

Notes. One species.

\section{Dunbrodya Jacoby, 1906}

References. Jacoby, 1906: 19.

Type species. Dunbrodya nitida Jacoby, 1906: 20 (Cape Colony), by monotypy.

Distribution. Republic of South Africa (Western Cape Province).

Ecology. The only species in this genus was collected on Asparagus sp. (Asparagaceae) (Jacoby, 1906).

Notes. One species.

[Embolimus Weise, 1902]

$=$ Decaria Weise, 1895

\section{[Entymosina Weise, 1910]}

=Abrarius Fairmaire, 1902

\section{Epitrix Foudras, 1860}

=Euplecnema Jacoby, 1906 (synonymized by Scherer, 1963)

References. Foudras, 1860: 147; Jacoby, 1906: 22; Scherer, 1963: 672.

Type species. Epitrix atropae Foudras, 1860: 55 (Europe), by subsequent designation by Maulik (1926: 130, 133).

Distribution. All zoogeographical regions.

Ecology. Genus mainly associated with Solanaceae. Some species can be harmful to plants of economic importance (cf. Jolivet \& Hawkeswood, 1995).

Notes. About a dozen species in Sub-Saharan Africa and Madagascar.

[Eremiella Weise, 1910]

=Eurylegna Weise, 1910

\section{Eriotica Harold, 1877}

=Niphraea Baly, 1878 (synonymized by Harold, 1878)

=Aridohespera Selman, 1963 (synonymized by Selman, 1968)

References. Harold, 1877a: 107; 1878: 206; Baly, 1878: 40; Ferreira, 1963: 516; Selman, 1963: 1156; 1968: 248.

Type species. Eriotica fuscipennis Harold 1877a: 107 (Nyassa), by monotypy.

Distribution. Ethiopia, Tanzania, Malawi and Mozambique.

Ecology. No information.

Notes. Seven species.

\section{[Escaleriella Weise, 1907]}

=Lypnea Baly, 1876

\section{Ethiopia Scherer, 1972}

References. Scherer, 1972: 7; 1978: 265.

Type species. Ethiopia tricolor Scherer, 1972: 7 (Ethiopia: Agheresalam), by original designation.

Distribution. Ethiopia and Yemen(?) (cf. Scherer, 1978)

Ecology. No information.

Notes. One species.

\section{Eugonotes Jacoby, 1897}

subgenus of Sanckia Duvivier, 1891
=Brancucciella Medvedev, 1995 syn. $\mathrm{n}$

Type species. Eugonotes longicornis Jacoby, 1897: 559 (Madagascar: Diego-Suarez), by monotypy.

References. Jacoby, 1897: 558; Laboissière, 1942: 15; Bechyné, 1956: 173; Medvedev, 1995: 479.

Distribution. Senegal(!), Guinea, Ethiopia, Democratic Republic of Congo, Uganda, Rwanda, Kenya, Madagascar, southern Indo-Malayan region.

Ecology. No information.

Notes. Five species in Sub-Saharan Africa and ten in Madagascar. The examination of the type material of Brancucciella micheli Medvedev (1995: 480) resulted in the following new synonymy: Eugonotes Jacoby, 1897 = Brancucciella Medvedev, 1995 syn. $n$.

[Euplecnema Jacoby, 1906]

=Epitrix Foudras, 1860

Eurylegna Weise, 1910

=Eremiella Weise, 1910 (synonymized by Scherer, 1972)

References. Weise, 1910a: 228; Scherer, 1972: 10.

Type species. Eurylegna fulva Weise, 1910a: 228 (Kilimanjaro), by monotypy.

Distribution. Ethiopia, Democratic Republic of Congo and Rwanda.

Ecology. No information.

Notes. Five species.

Eurylegniella Scherer, 1972

References. Scherer, 1972: 12.

Type species. Eurylegna guineensis Bechyné, 1955b: 528 (French Guinea: Mount Gangan; Dalaba; Nzérékoré; Mount Nimba), by original designation.

Distribution. Nigeria, Guinea and Democratic Republic of Congo (Garamba National Park).

Ecology. No information.

Notes. One species

[Eutheca Baly, 1878 nom. praeocc.]

See Calotheca Heyden, 1887 and Blepharidula Weise, 1916.

Eutornus Clark, 1860

Oedionychis Latreille, 1829 (pars)

References. Clark, 1860: 64; Bechyné, 1959:

Type species. Oedyionychus (Eutornus) africanus Clark, 1860: 65 , by original designation.

Distribution. Sub-Saharan Africa (absent in the northerneastern part of EAF) and Madagascar.

Ecology. No information.

Notes. About ten species, of which one is on Madagascar.

[Exorhina Weise, 1886]

=Chaetocnema Stephens, 1831

Gabonia Jacoby, 1893

=Thrymnes Weise, 1895 (synonymized by Weise, 1902a)

= Jamesonia Jacoby, 1895 (synonymized by Weise, 1902a)

=Orneates Jacoby, 1899 (synonymized by Weise, 1910a)

References. Jacoby, 1893: 101; 1895: 341; 1899b: 345; Weise, 1895: 338; 1902a: 173; 1910a: 231; Bechyné, 1955b: 489; Scherer, 1962a: 21; Scherer \& Boppré, 1997: 10, 32; Biondi \& D'Alessandro, 2003: 105.

Type species. Gabonia unicostata Jacoby, 1893: 101 (Gabon), by monotypy. 
Distribution. Afrotropical region (excluding Madagascar) and Arabian Peninsula(?).

Ecology. Polyphagous genus associated with several plant families (cf. Jolivet \& Hawkeswood, 1995; pers. data).

Notes. About one hundred and fifty species in Sub-Saharan Africa. According to Biondi \& D'Alessandro (2003), Gabonia is closely related to Luperomorpha Weise, 1887, a genus prevalent and widespread in the Oriental and Australian regions, and is probably a synonym. In fact, many species currently attributed to Gabonia do not show any significant differences from species of Luperomorpha. The diagnostic character reported by Scherer \& Boppré (1997) for separating these two genera is the apical spur on the hind tibiae, long and straight in Gabonia and very short in Luperomorpha, but this character is not always reliable. In view of the high variability shown by the genus Gabonia and the need to consider various other genera, synonymy with Luperomorpha may be confirmed in the future by a detailed and careful comparative study of this complicated African genus.

\section{[Gastrida Chapuis, 1879]}

Genus transferred to the subfamily Galerucinae (Furth \& Suzuki, 1994).

References. Chapuis, 1879: 20; Furth \& Suzuki, 1994: 131.

\section{[Graptodera Chevrolat, 1837]}

=Altica Geoffroy, 1762

\section{Guilelmia Weise, 1924}

References. Weise, 1924: 23; Scherer, 1961: 269 (sub Guilielmia); Biondi \& D'Alessandro, 2003: 97.

Type species. Guilelmia monticola Weise, 1924: 24 (Birunga, Mount Mukeno), by monotypy.

Distribution. Uganda and Rwanda.

Ecology. The only species in this genus was collected at a high altitude (3,100 $\mathrm{m}$ a.s.l.) (Weise, 1924).

Notes. One species.

\section{Guinerestia Scherer, 1959}

References. Scherer, 1959: 243-244; 1962a: 57.

Type species. Guinerestia rubripes Scherer, 1959: 244 (Nigeria-Cameroun: Mamfe), by original designation.

Distribution. Guinea, Nigeria, Democratic Republic of Congo and Rwanda(!).

Ecology. No information.

Notes. Three species.

\section{[Haltica Illiger, 1801]}

=Altica Geoffroy, 1762

[Halticella Jacoby, 1899 nom. praeocc.]

See Halticorthaea Csiki, 1940.

\section{Halticopsis Fairmaire, 1883}

References. Fairmaire, 1883a: 197; 1883b: 112.

Type species. Halticopsis spissicornis Fairmaire, 1883b: 112 (Mountains of Abyssinia), by monotypy.

Distribution. Ethiopia.

Ecology. No information.

Notes. One species.

\section{[Halticorthaea Csiki, 1940]}

=Amphimela Chapuis, 1875

References. Csiki in Heikertinger \& Csiki, 1940: 418; Jacoby, 1899b: 357.
Notes. New name proposed for Halticella Jacoby, 1899 preoccupied by Halticella Stephens, 1829: 36 (Hymenoptera, Chalcidoidea).

\section{Halticotropis Fairmaire, 1886}

References. Fairmaire, 1886: 95; Bechyné, 1964: 158.

Type species. Halticotropis multiplicata Fairmaire, 1886: 95 (Madagascar), by monotypy.

Distribution. Madagascar.

Ecology. No information.

Notes. Two species.

[Halticova Fairmaire, 1898]

=Amphimela Chapuis, 1875

[Haltitarsus Berthold, 1827]

=Dibolia Latreille, 1829

\section{Hemipyxis Chevrolat, 1837}

=Sebaethe Baly, 1864 (synonymized by Monrós \& Bechyné, 1956)

Asphaera Chevrolat, 1843 (pars)

References. Chevrolat, 1837: 411; 1843: 227; Baly, 1864: 438; Monrós \& Bechyné, 1956: 1134; Bechyné, 1958b: 193, 1960a: 110

Type species. Haltica troglodytes Olivier, 1808: 700 (India), by subsequent designation by Chevrolat (1845: 6).

Distribution. Afrotropical (including Madagascar), eastern Palaearctic, Indo-Malayan and Australian regions.

Ecology. Polyphagous genus associated with herbaceous plants and shrubs belonging to many plant families (cf. Jolivet \& Hawkeswood, 1995).

Notes. About thirty species in Sub-Saharan Africa and about ten in Madagascar. Bechyné (1959c) also attributed Asphaera brevicornis Jacoby (1892a: 573) to this genus.

\section{[Hermaeophaga Foudras, 1859]}

Not present in the Afrotropical region.

References. Foudras, 1859: 147.

Notes. To this Palaearctic genus were attributed species that were subsequently transferred to Orthocrepis Weise (cf. Scherer, 1961: 267).

\section{Hespera Weise, 1889}

=Allomorpha Jacoby, 1892 (synonymized by Maulik, 1926)

References. Weise, 1889: 638; Jacoby, 1892: 934; Maulik, 1926: 137).

Type species. Hespera sericea Weise, 1889: 639 (China), by original designation.

Distribution. Afrotropical (excluding Madagascar), eastern Palaeartic and Indo-Malayan regions.

Ecology. The Afrotropical species of this genus are mainly associated with Anacardiaceae and Ericaceae (cf. Jolivet \& Hawkeswood, 1995; pers. data).

Notes. About thirty species in Sub-Saharan Africa.

\section{Hildenbrandtina Weise, 1910}

Genus here transferred from the subfamily Galerucinae.

References. Weise, 1910b: 464; Laboissière, 1932: 58; Bechyné, 1948b: 99; 1964: 131.

Type species. Hildenbrandtina variegata Weise, 1910: 465 (Madagascar), by original designation.

Distribution. Madagascar.

Ecology. No information. 
Notes. This genus, with about ten species in Madagascar, is here transferred from Galerucinae to Alticinae because it has a metafemoral spring very similar to that described for the Indo-Malayan genus Mandarella Duvivier (1892: 433) (cf. Furth \& Suzuki, 1994).

\section{Homichloda Weise, 1902}

=Weiseana Jacoby, 1903 (synonymized by Cox, 1997)

References. Weise, 1902: 165; Jacoby, 1903a: 16; 1906: 23; Cox, 1997: 939.

Type species. Homichloda pauli Weise, 1902: 166 (Kwai), by monotypy.

Distribution. Kenya, Tanzania, Zambia and Republic of South Africa (KwaZulu-Natal).

Ecology. Genus associated with Mimosaceae (Acacia spp.) (Cox, 1997).

Notes. Three species.

\section{[Hyphasis Harold, 1877]}

Not present in the Afrotropical region.

References. Harold, 1877b: 434; Jacoby, 1901: 298; 1903b: 110; Maulik, 1926: 158, 166; Heikertinger \& Csiki, 1940: 457; Bechyné, 1948a: 10; 1958a: 90.

Notes. The Malagasy species initially attributed to this IndoMalayan genus were previously transferred to Hyphasoma Jacoby (1903b: 110) (Heikertinger \& Csiki, 1940) and then to Physoma Clark (Bechyné, 1948a). Bechyné (1958a) reports Hyphasoma sita Maulik (1926: 158), described from Sri Lanka (=Ceylon), as an introduced species in the Mascarene Islands (Mauritius).

[Jamesonia Jacoby, 1895]

=Gabonia Jacoby, 1893

\section{Kanonga Bechyné, 1960}

References. Bechyné, 1960a: 54; Biondi \& D’Alessandro, 2003: 104

Type species. Kanonga atra Bechyné, 1960a: 54 (Upemba National Park: Kanonga), by original designation.

Distribution. Democratic Republic of Congo and Togo(!).

Ecology. No information.

Notes. One species.

\section{Kenialtica Bechyné, 1960}

=Mediafra Scherer, 1961 (synonymy reported in Seeno \& Wilcox, 1982)

References. Bechyné, 1960a: 75; Scherer, 1961: 266; Seeno \& Wilcox, 1982: 136.

Type species. Aphthona muhavura Bechyné, 1955a: 207 (Rwanda, East of Muhavura), by original designation.

Distribution. Sierra Leone(!), Congo(!), Democratic Republic of Congo, Rwanda, Kenya(!), Republic of South Africa [Limpopo(!)] and Madagascar(!).

Ecology. No information.

Notes. Seven species.

\section{Kimongona Bechyné, 1959}

References. Bechyné, 1959a: 19.

Type species. Kimongona callifera Bechyné, 1959a: 19 (Belgian Congo: Mayumbe, Kimongo), by original designation.

Distribution. Democratic Republic of Congo and Rwanda(!).

Ecology. No information.

Notes. Two species.

\section{[Lactica Erichson, 1847]}

Not present in the Afrotropical region.

References. Erichson, 1847: 173; Weise, 1902b: 302; Bryant, 1940: 46; Bechyné, 1959a: 19.

Notes. The Afrotropical species initially attributed to this Neotropical genus were transferred to Phygasia Chapuis (Weise, 1902b; Bryant, 1940) and the Malagasy species to Antanemora Bechyné (Bechyné, 1959a).

\section{Lampedona Weise, 1907}

References. Weise, 1907a: 399.

Type species. Lampedona tarsalis Weise, 1907a: 399 (Spanish Guinea), by monotypy.

Distribution. Equatorial Guinea, Congo, Democratic Republic of Congo and Tanzania.

Ecology. This genus is associated with open-steppe habitats.

Notes. Three species.

\section{Lepialtica Scherer, 1962}

References. Scherer, 1962a: 30.

Type species. Lepialtica bicolor Scherer, 1962a: 31 (Garamba National Park), by monotypy.

Distribution. Democratic Republic of Congo.

Ecology. No information.

Notes. One species.

\section{[Livolia Jacoby, 1903]}

Genus transferred to the subfamily Galerucinae (Furth \& Suzuki, 1994).

References. Jacoby, 1903a: 16; Furth \& Suzuki, 1994: 132.

\section{Longitarsus Berthold, 1827}

References. Berthold, 1827: 401; Bechyné, 1958c: 8; 1960a: 55; Biondi \& D'Alessandro, 2008b: 179.

Type species. Chrysomela atricilla Linnaeus, 1761: 166 (Europe), by subsequent designation by Maulik (1926: 333).

Distribution. All zoogeographical regions.

Ecology. Polyphagous genus associated with several plant families, in particular Boraginaceae, Asteraceae, Lamiaceae and Scrophulariaceae (cf. Jolivet \& Hawkeswood, 1995; pers. data).

Notes. Over one hundred species in Sub-Saharan Africa and Madagascar (pers. data).

\section{Luperomorpha Weise, 1887}

References. Weise, 1887: 202; 1915: 179; Bechyné, 1959a: 1; Doguet, 1979: 308 .

Type species. Luperomorpha trivialis Weise, 1887: 204 (Siberia: Raddefka; Chingan), by original designation.

Distribution. Nigeria, Cameroon, Equatorial Guinea, and Democratic Republic of Congo, Arabian Peninsula, and eastern Palaearctic, Indo-Malayan, and Australian regions.

Ecology. Polyphagous (cf. Jolivet \& Hawkeswood, 1995), but no information for the Afrotropical region.

Notes. Only one species in the Afrotropical region: Luperomorpha vittula (Weise, 1915) [described under Jamesonia, transferred to Luperomorpha by Bechyné (1959)].

\section{Lypnea Baly, 1876}

=Escaleriella Weise, 1907 (synonymized by Scherer, 1961)

=Poephila Weise, 1895 [nec Poephila Gould, 1842: 93 (pl.), Aves, Estrildidae]

=Poephilina Csiki, 1940 (new name for Poephila Weise, 1895; synonymized by Bechyné, 1968) 
References. Baly, 1876a: 446; Weise, 1895: 342; 1907a: 396; Csiki in Heikertinger \& Csiki, 1940: 349; Bechyné, 1960b: 15; 1968: 1717-1718; Scherer, 1961: 267.

Type species. Lypnea flava Baly, 1876a: 446 (New Guinea, Batchian), by monotypy.

Distribution. Afrotropical [including Madagascar (1)], eastern Palaearctic, Indo-Malayan and Australian regions.

Ecology. Lypnea flaveola (Bryant, 1944b: 141) was collected on Oncoba echinata Oliver (Flacourtiaceae) (Bryant, 1944b, sub Poephila).

Notes. About ten species in Sub-Saharan Africa and Madagascar. Bechyné (1968: 1718) considered Escaleriella Weise and Lypnea Baly to be separate genera because of the different shape of their elytral epipleura: expanded in Lypnea; straight and narrow in Escaleriella.

\section{[Macroorthocrepis Pic, 1921]}

=Phygasia Chevrolat, 1837

\section{Malvernia Jacoby, 1899}

References. Jacoby, 1899b: 346; Biondi, 1998a: 37; Biondi \& D'Alessandro, 2003: 104.

Type species. Malvernia varicornis Jacoby, 1899b: 347 (KwaZulu-Natal, Malvern), by monotypy.

Distribution. Malawi(!) and Republic of South Africa (Limpopo, North-West Province, Gauteng, Mpumalanga, Free State, KwaZulu-Natal, Eastern Cape Province).

Ecology. Malvernia varicornis was collected on flowers of Burchellia bubalina (L. f.) Simms (Rubiaceae) (Biondi, 1998a).

Notes. Two species.

\section{Manobia Jacoby, 1885}

=Afroalytus Scherer, 1961 (synonymized by Biondi, 2001)

References. Jacoby, 1885: 73; Scherer, 1961: 269; 1962a: 54; Biondi, 2001: 648.

Type species. Manobia nigripennis Jacoby, 1885: 73 (Sumatra), by subsequent designation by Maulik (1926: 285, 407).

Distribution. Western and central Africa; eastern Palaearctic, Indo-Malayan and Australian regions.

Ecology. Genus probably polyphagous, reported on Epacridaceae, Urticaceae, Cyatheaceae, Asteraceae, Arecaceae, etc. (cf. Jolivet \& Hawkeswood, 1995).

Notes. About fifteen species in Sub-Saharan Africa.

\section{[Mantura Stephens, 1831]}

Not present in the Afrotropical region.

=Balanomorpha Chevrolat, 1837 (synonymy reported in Heikertinger \& Csiki, 1940)

References. Stephens, 1831: 285, 322; Chevrolat, 1837: 417; Weise, 1907: 222; Bechyné, 1955a: 220.

Notes. Mantura quadriplagiata Jacoby (1895: 321) transferred by Bechyné (1955a) to Podagrica Chevrolat; Balanomorpha aethiopica Chapuis (1879: 13) transferred by Weise (1907) to Neumannia Weise, 1907 nom. praeocc. (=Podagricina Csiki, 1940).

\section{[Mediafra Scherer, 1961]}

=Kenialtica Bechyné, 1960

\section{Mesocrepis Scherer, 1963}

References. Scherer, 1963: 668.

Type species. Mesocrepis lindemannae Scherer, 1963: 669 (Tanzania: Njombe), by original designation.

Distribution. Tanzania and Republic of South Africa [Mpumalanga(!)].
Ecology. No information.

Notes. One species.

\section{Metroserrapha Bechyné, 1958}

References. Bechyné 1958a: 86; Doguet, 1974: 120.

Type species. Metroserrapha prima Bechyné, 1958a: 86 (Mauritius Island), by original designation.

Distribution. Mascarene Islands and Madagascar(!).

Ecology. Genus probably polyphagous associated with Ericaceae, Asteraceae, Polygonaceae, etc. (cf. Jolivet \& Hawkeswood, 1995).

Notes. Seven species on the Mascarene Islands and about ten, not yet described, on Madagascar (pers. data).

\section{[Monodaltica Bechyné, 1955]}

=Trachytetra Sharp, 1886

\section{Montiaphthona Scherer, 1961}

References. Scherer, 1961: 282; 1962a: 17; Biondi \& D'Alessandro, 2003: 98.

Type species. Montiaphthona monticola Scherer, 1961: 285 (Kivu: Mont Muhi), by original designation.

Distribution. Democratic Republic of Congo, Rwanda, Uganda(!), Kenya and Tanzania.

Ecology. The species of this genus generally live at altitudes above $2,500 \mathrm{~m}$ in mixed bamboo forests.

Notes. Six species.

[Musaka Bechyné, 1958]

=Sphaeroderma Stephens, 1831

\section{Myrcina Chapuis, 1875}

=Xenaltica Baly, 1875 (synonymized by Laboissière, 1942)

=Myrcinella Jacoby, 1901 (synonymized by Bechyné, 1964)

References. Chapuis,1875: 124, 126; Baly, 1875: 25; Jacoby, 1901: 301; Laboissière, 1942: 50; Bechyné, 1964: 150).

Type species. Myrcina nigra Chapuis, 1875: 127 (VieuxCalabar), by original designation.

Distribution. Sub-Saharan Africa (absent in the southern part of SAF) and Madagascar.

Ecology. Some species of this genus were collected on Spathodea sp. (Bignoniaceae) in eastern Africa (cf. Jolivet \& Hawkeswood, 1995).

Notes. Twenty-three species.

[Myrcinella Jacoby, 1901]

=Myrcina Chapuis, 1875

\section{Neodera Duvivier, 1891}

includes subgen. Neoderina Bechyné, 1952

References. Duvivier, 1891: 316; Bechyné, 1947b: 139; 1952: 251; 1964: 152;

Type species. Crepidodera picticornis Harold, 1877: 107 (Madagascar), by original designation.

Distribution. Madagascar. Weise (1923: 122) described Neodera australis from Australia (Queensland): however the generic attribution of this species needs further confirmation.

Ecology. No information.

Notes. About fifteen species. The subgenus Neoderina Bechyné, 1952 could be a synonym of Diphaulacosoma Jacoby, 1892 (pers. data).

\section{Neoderina Bechyné, 1952}

subgenus of Neodera Duvivier, 1891

References. Bechyné, 1952: 251. 
Type species. Neodera crassicornis Bechyné, 1952: 251 (Madagascar: Ambohitsitondrona) by monotypy.

Distribution. Northeastern Madagascar.

Ecology. No information.

Notes. One species. Neoderina may be a synonym of Diphaulacosoma Jacoby, 1892 (pers. data).

\section{[Neumannia Weise, 1907 nom. praeocc.]}

See Podagricina Csiki, 1940.

[Niphraea Baly, 1878]

$=$ Eriotica Harold, 1877

\section{Nisotra Baly, 1864}

=Pseudonisotra Bechyné, 1968 syn. $\mathrm{n}$.

References. Baly, 1864: 437; Bechyné, 1955a: 220; 1959d: 153; 1960a: 88; 1968: 1719.

Type species. Haltica gemella Erichson, 1834: 275 (Philippines: Luzon), by subsequent designation by Chapuis (1875: 42).

Distribution. Afrotropical (including Madagascar), IndoMalayan and Australian regions.

Ecology. Genus mainly associated with Malvaceae (cf. Jolivet \& Hawkeswood, 1995).

Notes. About seventy species of this genus occur in SubSaharan Africa and Madagascar.There are no important diagnostic characters distinguishing Pseudonisotra Bechyné from Nisotra. Therefore, the following new synonymy is proposed: Nisotra Baly, 1864 = Pseudonisotra Bechyné, 1968 syn. n.

\section{Notomela Jacoby, 1899}

References. Jacoby, 1899b: 357; Bryant, 1931: 255; Scherer, 1969: 371.

Type species. Notomela cyanipennis Jacoby, 1899b: 357 (Cameroun), by monotypy.

Distribution. Liberia, Ivory Coast, Nigeria, Cameroon, Equatorial Guinea (Fernando Poo Island), Uganda, Rwanda(!), Democratic Republic of Congo, and Republic of South Africa (North-West Province and KwaZulu-Natal).

Ecology. Notomela fulvicollis Bryant, 1931 collected on Xanthoxylon capense Harv. (Rutaceae) in southeastern Africa (Bryant, 1931).

Notes. Four species.

\section{Nzerekorena Bechyné, 1955}

References. Bechyné, 1955b: 507; Scherer, 1959: 190; Scherer \& Boppré, 1997: 10, 32; Biondi \& D’Alessandro, 2003: 104.

Type species. Nzerekorena cerambycina Bechyné, 1955b: 507 (French Guinea: Nzérékoré; Liberia: Kaouyéké; Dahomey: Forèt de Ketou), by original designation.

Distribution. Guinea, Liberia, Benin, Nigeria, Cameroon, Democratic Republic of Congo, Kenya and Uganda(!).

Ecology. No information.

Notes. Nine species.

\section{[Ochrosis Foudras, 1860]}

Not present in the Afrotropical region.

References. Foudras, 1860: 147; Jacoby, 1906: 17.

Notes. Ochrosis natalensis Jacoby (1906: 17) was attributed to this Palaearctic genus. The examination of the type material resulted in the transfer of this species to Bechuana as Bechuana natalensis (Jacoby, 1906) comb. $\mathrm{n}$.

[Oedionychis Latreille, 1829]

Not present in the Afrotropical region.
References. Latreille, 1829: 154; Konstantinov \& Vandenberg, 1996: 367.

Notes. Currently, only four species that occur in southwestern Europe and North Africa are attributed to this genus (Konstantinov \& Vandenberg, 1996). Afrotropical species described as Oedionychis were previously transferred to the genera Eutornus Clark, Philopona Weise, Physodactyla Chapuis, Physoma Clark, and Physomandroya Bechyné.

[Orneates Jacoby, 1899]

=Gabonia Jacoby, 1893

\section{Orthocrepis Weise, 1888}

Hermaeophaga Foudras, 1859 (pars)

Crepidodera Chevrolat, 1837 (pars)

References. Weise, 1888: 850; Bechyné, 1948a: 4 (sub Hermaeophaga); 1954b: 677; 1955a: 224; 1964: 141; Scherer, 1961: 267; 1963: 664.

Type species. Haltica ruficollis Lucas, 1849: 546 (Algeria), by monotypy.

Distribution. Afrotropical (including Madagascar), western Palaearctic and Indo-Malayan regions.

Ecology. Genus mainly associated with Euphorbiaceae, but also with Leguminosae and Malvaceae (cf. Jolivet \& Hawkeswood, 1995; Pollard, 1957).

Notes. About twenty-five species in Sub-Saharan Africa and sixteen on Madagascar.

\section{Paradibolia Baly, 1875}

References. Baly, 1875: 31; Weise, 1912: 157; Bryant, 1927: 617.

Type species. Paradibolia indica Baly, 1875: 31 (India), by monotypy.

Distribution. Guinea, Sierra Leone, Cameroon, Democratic Republic of Congo, Namibia(!), Republic of South Africa, and Indo-Malayan and Australian regions.

Ecology. Genus associated with Lamiaceae in South Africa (pers. data).

Notes. Three species.

\section{Paropsiderma Bechyné, 1958}

References. Bechyné, 1958a: 92.

Type species. Sphaeroderma anthrax Brancsik, 1891: 151 (Madagascar), by monotypy.

Distribution. Madagascar.

Ecology. No information.

Notes. Three species.

\section{Perichilona Weise, 1919}

References. Weise, 1919: 202.

Type species. Perichilona rufa Weise, 1919: 203 (Gaviro, Kwiro), by original designation.

Distribution. Tanzania.

Ecology. No information.

Notes. Two species.

\section{Philopona Weise, 1903}

Oedionychis Latreille, 1829 (pars)

References. Weise, 1903: 216; Laboissière, 1942: 105.

Type species. Oedionychis (?) vernicata Gerstaecker, 1871: 84 (Zanzibar), by original designation.

Distribution. Afrotropical (excluding Madagascar), southeastern Palaearctic, Indo-Malayan and Australian regions.

Ecology. P. usambarica Csiki (in Heikertinger \& Csiki, 1940: 453) was collected on Thunbergia alata Bojer ex Simms (Acanthaceae) in Kenya (Furth, 1985). 
Notes. About twenty species in Sub-Saharan Africa.

Phygasia Chevrolat, 1837

=Macroorthocrepis Pic, 1921 (synonymized by Bechyné, 1960a)

Lactica Erichson, 1847 (pars)

References. Chevrolat, 1837: 411; Harold, 1877: 365; Fairmaire, 1888: 156; Pic, 1921: 14; Bryant, 1940: 46; Bechyné, 1952: 249; 1960a: 82.

Type species. Altica unicolor Olivier, 1808: 699 (India), by original designation.

Distribution. Afrotropical (including Madagascar), eastern Palaearctic and Indo-Malayan regions.

Ecology. Genus mainly associated with Asclepiadaceae (cf. Jolivet \& Hawkeswood, 1995).

Notes. About forty species in Sub-Saharan Africa and Madagascar.

\section{Phyllotreta Chevrolat, 1837}

References. Chevrolat, 1837: 415; Bryant, 1942a: 145; Bechyné, 1955c: 61.

Type species. Chrysomela brassicae Fabricius, 1787: 78 (Europe), by subsequent designation by Chevrolat (1845: 6).

Distribution. All zoogeographical regions.

Ecology. Genus mainly associated with Cruciferae, Resedaceae, and Capparidaceae (cf. Jolivet \& Hawkeswood, 1995).

Notes. About forty species in Sub-Saharan Africa, Arabian Peninsula and Madagascar.

\section{Physodactyla Chapuis, 1875}

Oedionychis Latreille, 1829 (pars)

References. Chapuis, 1875: 83, 88; Scherer 1962a: 72.

Type species. Physonychis africana Chapuis, 1875: 89 (East Africa), by original designation.

Distribution. Sudan, Democratic Republic of Congo, Kenya, Tanzania and Republic of South Africa (Mpumalanga and KwaZulu-Natal).

Ecology. P. rubiginosa (Gerstaecker, 1871: 84) was collected on Thunbergia alata Bojer ex Simms (Acanthaceae) in Kenya (Furth, 1985); P. africana (Chapuis) on Digera arvensis Forssk. (Amaranthaceae) in Sudan (Pollard, 1957, sub Physonychis).

Notes. Six species.

\section{Physoma Clark, 1863}

=Tropidophora Thomson, 1858: 217 (unavailable; synonymized by Jacoby, 1888)

Hyphasys Harold, 1877 (pars)

Hyphasoma Jacoby, 1903 (pars)

Oedionychis Latreille, 1829 (pars)

References. Clark, 1863: 165; Thomson, 1858: 217; Chapuis, 1875: 83, 87; Harold, 1877b: 434; Weise, 1895: 344; Jacoby, 1888: 205; 1903b: 110; Bechyné, 1959c: 318; Scherer, 1962: 73 (sub Physonychis).

Type species. Physoma tripartitum (Thomson, 1858) (Gabon) (=Physonychis rugicollis Clark, 1860 i.1.), by subsequent designation by Chapuis (1875).

Distribution. Western and central Africa and Madagascar.

Ecology. No information.

Notes. Two species in Sub-Saharan Africa and about twenty in Madagascar. The generic name Tropidophora Thomson is not available because it was ambiguously applied (ICZN, 1999: art. 12.2.5).

\section{Physomandroya Bechyné, 1959}

Asphaera Chevrolat, 1843 (pars)

Oedionychis Latreille, 1829 (pars)

References. Chevrolat, 1843: 227; Bechyné, 1959c: 318.

Type species. Physomandroya decorsei Bechyné, 1959c: 319

(Madagascar: Ambowombé), by original designation.

Distribution. Madagascar.

Ecology. No information.

Notes. Seven species. Bechyné (1959c) also transferred to this genus Asphaera melanarthra Fairmaire, 1886: 94 (=Asphaera madagascariensis Jacoby, 1892a: 573).

\section{Physonychis Clark, 1860}

References. Clark, 1860: 29; Duvivier, 1891: 424; Bechyné, 1959c: 321.

Type species. Physonychis smaragdina Clark, 1860: 31 (Western Africa), by original designation.

Distribution. Sub-Saharan Africa (absent in the southernwestern part of SAF and Madagascar). Physonychis varicornis Duvivier, 1891 from Madagascar was transferred to the genus Physoma Clark by Bechyné (1959c).

Ecology. No information.

Notes. About thirty species.

[Plectroscelis Chevrolat, 1837]

=Chaetocnema Stephens, 1831

\section{Podagrica Chevrolat, 1837}

=Podagrixena Bechyné, 1968 syn. n.

References. Chevrolat, 1837: 418; Bryant, 1942b: 229; Bechyné, 1960a: 84; 1968: 1719.

Type species. Altica fuscipes Fabricius, 1775: 114 (Europe), by subsequent designation by Maulik, (1926: 273).

Distribution. Afrotropical (excluding Madagascar), Palaearctic and Indo-Malayan regions.

Ecology. Genus mainly associated with Malvaceae (cf. Jolivet \& Hawkeswood, 1995: 128). Some species can damage cotton crops, Gossypium sp. (Malvaceae).

Notes. About fifty species in Sub-Saharan Africa. There are no important diagnostic characters distinguishing Podagrixena Bechyné from Podagrica. Therefore, the following new synonymy is proposed: Podagrica Chevrolat, $1837=$ Podagrixena Bechyné, 1968 syn. n.

\section{Podagricina Csiki, 1940}

=Neumannia Weise, 1907 (nec Neumania Lebert, 1879: 357, Acari, Unionicolidae)

References. Csiki in Heikertinger \& Csiki, 1940: 364; Weise, 1907b: 223; Bechyné, 1960a: 88.

Type species. Balanomorpha aethiopica Chapuis, 1879: 13 (Ethiopia, Abyssinia), by original designation.

Distribution. Ethiopia, Uganda and Tanzania.

Ecology. No information.

Notes. Three species. New name proposed for Neumannia Weise, 1907 preoccupied by Neumania Lebert, 1879: 357 (Acari, Unionicolidae).

\section{[Podagrixena Bechyné, 1968]}

=Podagrica Chevrolat, 1837

[Poephila Weise, 1895 nom. praeocc.]

See Poephilina Csiki, 1940.

[Poephilina Csiki, 1940]

=Lypnea Baly, 1876 
References. Csiki in Heikertinger \& Csiki, 1940: 349; Weise, 1895: 342 .

Notes. New name proposed for Poephila Weise preoccupied by Poephila Gould (1842: 93 [pl.]) (Aves, Estrildidae).

\section{Polyclada Chevrolat, 1835}

$=$ Cladocera Hope, 1840 (synonymy reported in Achard, 1922)

=Cladotelia Kolbe, 1894 (synonymy reported in Achard, 1922)

References. Chevrolat, 1835: 375; Hope, 1840: 169; Baly, 1861: 198; Kolbe, 1894: 86; Achard, 1922: 4; Medvedev, 1996: 261.

Type species. Clythra pectinicornis Olivier, 1789: 31 (Africa), by monotypy.

Distribution. Sub-Saharan Africa (absent in the southwestern part of SAF and Madagascar), Saudi Arabia and Yemen.

Ecology. Genus mainly associated with Anarcadiaceae (cf. Jolivet \& Hawkeswood, 1995; Chaboo et al., 2007).

Notes. Sixteen species.

\section{Pratima Maulik, 1931}

References. Maulik 1931: 253; Bechyné, 1958a: 84.

Type species. Pratima variabilis Maulik, 1931: 253 (Seychelles: Silhouette and Mahe), by original designation.

Distribution. Indian Ocean (Seychelles and Mascarene Islands).

Ecology. The species of this genus live in forests $(300-700 \mathrm{~m}$ a.s.1.) (Maulik 1931).

Notes. Eight species

\section{[Prototrigona Chevrolat, 1837]}

Nomen nudum.

Notes. Chevrolat (1837) uses this generic name, without any description, for the following two never published species: "Glauca. Dej." synonymized with "Prasinipennis. Chevr." (also never published) from Madagascar, and "Viridana. Dej." from an unknown locality.

\section{Pseudadorium Fairmaire, 1885}

References. Fairmaire, 1885: 239; Bechyné, 1958b: 193.

Type species. Pseudadorium vernicatum Fairmaire, 1884: 239 (Madagascar), by monotypy.

Distribution. Madagascar.

Ecology. No information.

Notes. Five species.

\section{[Pseudonisotra Bechyné, 1968]}

=Nisotra Baly, 1864

\section{Psylliodes Berthold, 1827}

References. Berthold, 1827: 401; Biondi, 1996: 257; Nadein, 2007: 317.

Type species. Chrysomela chrysocephala Linnaeus, 1758: 372 (Europe), by subsequent designation by Maulik (1926: 144).

Distribution. All zoogeographical regions. In the Afrotropical region this genus is known from Ethiopia, Kenya, Tanzania and Republic of South Africa.

Ecology. Genus mainly associated with Cruciferae, Solanaceae and Graminae (cf. Jolivet \& Hawkeswood, 1995).

Notes. Seven species.

\section{Pydaristes Harold, 1875}

References. Harold, 1875: 446; Scherer, 1961: 252.

Type species. Pydaristes attagenoides Harold, 1875: 447 (Africa), by monotypy.

Distribution. "Africa".

Ecology. No information.
Notes. One species. Unfortunately, the location of the type material is unknown. The original description of Pydaristes seems to be identical or very close to that of Amphimela Chapuis. The synonymy proposed by Scherer (1961) between Pydaristes and Blepharida Chevrolat is unconvincing because of the absence in Pydaristes (by the original description) of dentiform emargination on hind tibiae, characteristically present in Blepharida.

\section{Sanckia Duvivier, 1891}

includes subgen. Eugonotes Jacoby, 1897 = Brancucciella Medvedev, 1995 syn. n. (see under Eugonotes).

References. Duvivier, 1891: 316; Jacoby, 1897: 558; Bechyné, 1956: 173; Medvedev, 1995: 479.

Type species. Sanckia johanna Duvivier, 1891: 316 (Madagascar: Antsianaka Forest), by original designation.

Distribution. Senegal(!), Guinea, Ethiopia, Democratic Republic of Congo, Uganda, Rwanda, Kenya and southern IndoMalayan region (subg. Eugonotes); Madagascar (both subgenera).

Ecology. No information.

Notes. About twenty species in the Afrotropical region, mostly in Madagascar.

\section{Serraphula Jacoby, 1897}

References. Jacoby, 1897: 556; Maulik, 1929: 208; Biondi \& D'Alessandro, 2010: 3.

Type species. Serraphula aenea Jacoby, 1897: 557 (Mashonaland), by monotypy.

Distribution. Zimbabwe and Republic of South Africa (Limpopo, Mpumalanga, Free State, KwaZulu-Natal, Eastern and Western Cape Provinces)

Ecology. Genus associated with Asteraceae (Biondi \& D'Alessandro, 2010).

Notes. Nineteen species.

\section{Sesquiphaera Bechyné, 1958}

References. Bechyné, 1958a: 92; Scherer, 1961: 275.

Type species. Sphaeroderma mashonanum Jacoby, 1900: 252 (Mashonaland: Salisbury), by original designation.

Distribution. Guinea Bissau(!), Guinea, Democratic Republic of Congo, Rwanda, Tanzania, Namibia(!), Zimbabwe and Republic of South Africa (Gauteng, Mpumalanga, KwaZuluNatal).

Ecology. No information.

Notes. Six species. Sphaeroderma natalensis Bryant (1943: 493) is here transferred to this genus as Sesquiphaera natalensis (Bryant, 1943) comb. $\mathrm{n}$.

\section{Seychellaltica Biondi, 2002}

References. Biondi, 2002b: 358 .

Type species. Chaetocnema mahensis Maulik, 1931: 250 (Seychelles: Mahé), by original designation.

Distribution. Indian Ocean: Seychelles.

Ecology. The species in this genus are associated with indigenous forests in Seychelles.

Notes. Four species.

Sjoestedtinia Weise, 1910

References. Weise, 1910a: 205; Bryant, 1953: 162; Scherer, 1963: 648

Type species. Sjoestedtinia montivaga Weise, 1910a: 206 (Kilimanjaro: Kibocho), by original designation.

Distribution. Uganda, Kenya(!), and Tanzania.

Ecology. This genus lives at high altitudes on Kilimanjaro and Mount Elgon. S. montivaga was collected on Lobelia deckeni 
(Asch.) Hemsl. (Campanulaceae) (Weise, 1910a), and S. fordi Bryant, 1953 on Senecio sp. (Asteraceae) in Uganda (Bryant, 1953) and on Lobelia sp. in Kenya (S. Zoia, pers. comm.).

Notes. Two species.

\section{Sphaeroderma Stephens, 1831}

=Argosomus Wollaston, 1867 (synonymized by Scherer, 1961)

=Musaka Bechyné, 1958 (synonymized by Scherer, 1961)

References. Stephens, 1831: 328; Wollaston, 1867: 152; Bryant, 1943: 487; Bechyné, 1958a: 89; 1968: 1702; Scherer, 1961: 252.

Type species. Altica testacea Fabricius, 1775: 114 (Europe), by subsequent designation by Maulik, (1926: 316).

Distribution. Afrotropical (including Madagascar), Palaearctic, Indo-Malayan and Australian regions. The species of Sphaeroderma reported from the Neotropical and Nearctic regions should be attributed to different genera (cf. Savini \& Furth, 2001).

Ecology. Genus mainly associated with Asteraceae and Ranunculaceae (cf. Jolivet \& Hawkeswood, 1995).

Notes. Over fifty species in Sub-Saharan African and about ten in Madagascar. Sphaeroderma natalensis Bryant (1943: 493) is here transferred to Sesquiphaera Bechyné, 1958 as Sesquiphaera natalensis (Bryant, 1943) comb. $\mathrm{n}$.

Sphaerophysa Baly, 1876

References. Baly, 1876b: 581; Bechyné, 1958c: 10.

Type species. Sphaerophysa clavicornis Baly, 1876b: 582 (Madagascar), by monotypy.

Distribution. Madagascar. Sphaerophysa piceicollis Jacoby (1889: 195) from Myanmar (=Burma), previously doubtfully attributed to this genus, was subsequently made the typespecies of the genus Jacobyana Maulik (1926: 302).

Ecology. No information.

Notes. Five species. Weise (1910: 496) wrongly established the synonymy between Sphaerophysa Baly, 1876 and Dibolosoma Jacoby, 1897.

\section{Stegnaspea Baly, 1877}

References. Baly, 1877a: 181.

Type species. Stegnaspea trimeni Baly, 1877a: 182 (Cape of Good Hope), by monotypy.

Distribution. Republic of South Africa (Western and Eastern Cape Provinces) and Tristan da Cunha(!).

Ecology. Stegnaspea trimeni was collected on Graminae in meadows (pers. data).

Notes. One species.

\section{Stuckenbergiana Scherer, 1963}

References. Scherer, 1963: 670.

Type species. Podagrica glabrata Jacoby, 1899b: 349 (KwaZulu-Natal: Umtenweni River; Eastern Cape Province: Port St. John), by original designation.

Distribution. Republic of South Africa [Mpumalanga(!), KwaZulu-Natal and Eastern Cape Province].

Ecology. No information.

Notes. One species.

\section{Terpnochlorus Fairmaire, 1904}

Chaloenus Westwood, 1862 (pars)

References. Fairmaire, 1904: 269; Laboissière, 1932: 575; Bryant, 1927: 615; Bechyné, 1955b: 543; 1960a: 101; Furth \& Suzuki, 1994: 130; Biondi, 2002b: 364.

Type species. Terpnochlorus perrieri Fairmaire, 1904: 269 (Madagascar: Soalala), by monotypy.

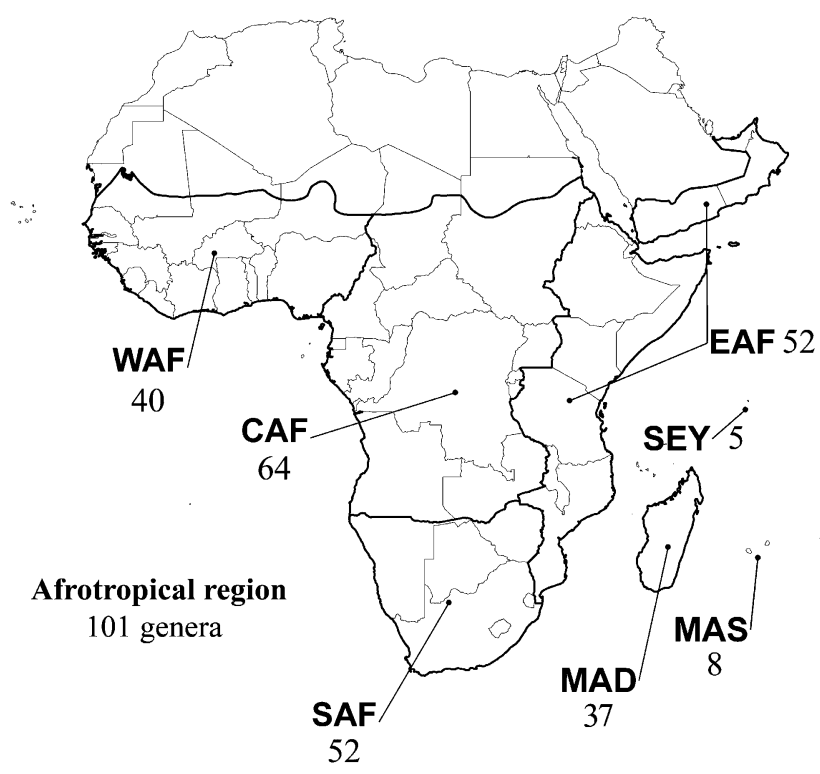

Fig. 2. Number of flea beetle genera occuring in the various areas of the Afrotropical region (see Material and methods for abbreviations) (modified from Graf \& Cummings, 2007).

Distribution. Mali, Gambia(!), Guinea Bissau(!), Sierra Leone, Democratic Republic of Congo, Botswana(!), Namibia(!), Madagascar and South America (Venezuela and Mexico).

Ecology. This genus lives in moist habitats generally associated with Juncaceae (Biondi, 2002b).

Notes. Two species in the Afrotropical region. Chaloenus viridis Bryant, 1927 = Terpnochlorus perrieri Fairmaire, 1904 (cf. Bechynè, 1955b).

\section{Torodera Weise, 1902}

References. Weise, 1902a: 163; Scherer, 1987: 67; Biondi, 1994: 437.

Type species. Torodera octomaculata Weise, 1902: 164 (Kwai), by subsequent designation by Scherer (1987: 67).

Distribution. Guinea, Sierra Leone, Sudan, Congo, Democratic Republic of Congo, Uganda, Kenya, Tanzania, Zimbabwe, Republic of South Africa (KwaZulu-Natal) and Indo-Malayan region.

Ecology. Genus reported on Graminae (Oryza) in Kenya (cf. Jolivet \& Hawkeswood, 1995).

Notes. Four species in the Afrotropical region.

\section{Toxaria Weise, 1903}

References. Weise, 1903: 215; 1912: 157; Laboissière, 1941: 318; Bryant, 1943: 488.

Type species. Galleruca indica Fabricius, 1798: 98 (Western Cape), by original designation.

Distribution. Democratic Republic of Congo, Uganda, Kenya and Republic of South Africa.

Ecology. No information.

Notes. Five species.

\section{Trachytetra Sharp, 1886}

= Monodaltica Bechyné, 1955 (synonymized by Konstantinov \& Prathapan, 2008)

References. Sharp, 1886: 449; Bechyné, 1955b: 509; Scherer, 1962a: 18 (sub Monodaltica); Konstantinov \& Prathapan, 2008: 413.

Type species. Phyllotreta rugulosa Broun, 1880: 636 (New Zealand), by original designation. 
TABLE 1. Occurrence of Alticinae genera of the Chrysomelidae in different areas of the Afrotropical region and other zoogeographical areas (see Material and methods for abbreviations).

\begin{tabular}{|c|c|c|c|c|c|c|c|c|c|c|c|c|}
\hline & WAF & CAF & EAF & SAF & MAD & SEY & MAS & Palaearctic & Nearctic & Neotropical I & Indo-Malayan & Australian \\
\hline Abrarius & & & & & $\mathrm{X}$ & & & & & $?$ & & \\
\hline Afroaltica & & & & $\mathrm{X}$ & & & & & & & & \\
\hline Afrocrepis & & & & $\mathrm{X}$ & & & & & & & & \\
\hline Afrorestia & & $X$ & $X$ & $\mathrm{X}$ & $\mathrm{X}$ & & & & & & & \\
\hline Alocypha & & & $\mathrm{X}$ & $X$ & & & & & & & & \\
\hline Altica & $\mathrm{X}$ & $X$ & $\mathrm{X}$ & $X$ & $\mathrm{X}$ & & $\mathrm{X}$ & $\mathrm{X}$ & $\mathrm{X}$ & $X$ & $\mathrm{X}$ & $X$ \\
\hline Amphimela & $\mathrm{X}$ & $\mathrm{X}$ & $\mathrm{X}$ & $\mathrm{X}$ & $\mathrm{X}$ & & & & & & $\mathrm{X}$ & $\mathrm{X}$ \\
\hline Anaxerta & & & & & $\mathrm{X}$ & & & & & & & \\
\hline Angulaphthona & $\mathrm{X}$ & $\mathrm{X}$ & $\mathrm{X}$ & & $X$ & & & $\mathrm{X}$ & & & & \\
\hline Antanemora & & & & & $\mathrm{X}$ & & & & & & & \\
\hline Aphthona & $\mathrm{X}$ & $X$ & $\mathrm{X}$ & $\mathrm{X}$ & $X$ & & $\mathrm{X}$ & $X$ & & & $X$ & $\mathrm{X}$ \\
\hline Argopistes & & $\mathrm{X}$ & $\mathrm{X}$ & $\mathrm{X}$ & $\mathrm{X}$ & & & $\mathrm{X}$ & $\mathrm{X}$ & $\mathrm{X}$ & $\mathrm{X}$ & $\mathrm{X}$ \\
\hline Bangalaltica & & $\mathrm{X}$ & & & & & & & & & & \\
\hline Bechuana & & & & $\mathrm{X}$ & & & & & & & & \\
\hline Bechynella & $\mathrm{X}$ & $\mathrm{X}$ & & & & & & & & & & \\
\hline Bikasha & & & & & & $\mathrm{X}$ & & & & & $\mathrm{X}$ & \\
\hline Biodontocnema & & & & $\mathrm{X}$ & & & & & & & & \\
\hline Blepharida & $\mathrm{X}$ & $\mathrm{X}$ & $\mathrm{X}$ & $\mathrm{X}$ & $\mathrm{X}$ & & & $X$ & $X$ & $\mathrm{X}$ & & \\
\hline Buphonella & & $X$ & $X$ & & & & & & & & & \\
\hline Carcharodis & & $\mathrm{X}$ & & $\mathrm{X}$ & $\mathrm{X}$ & & & & & & & \\
\hline Celisaltica & & $X$ & & & & & & & & & & \\
\hline Chaetocnema & $\mathrm{X}$ & $X$ & $\mathrm{X}$ & $\mathrm{X}$ & $\mathrm{X}$ & $\mathrm{X}$ & $\mathrm{X}$ & $\mathrm{X}$ & $X$ & $\mathrm{X}$ & $\mathrm{X}$ & $\mathrm{X}$ \\
\hline Chaillucola & & $\mathrm{X}$ & & & & & & & & & & \\
\hline Chirodica & & & & $\mathrm{X}$ & & & & & & & & \\
\hline Collartaltica & $\mathrm{X}$ & $\mathrm{X}$ & $\mathrm{X}$ & $\mathrm{X}$ & & & & & & & & \\
\hline Decaria & $\mathrm{X}$ & $X$ & $\mathrm{X}$ & $\mathrm{X}$ & & & & & & & & \\
\hline Diamphidia & & $\mathrm{X}$ & $\mathrm{X}$ & $\mathrm{X}$ & & & & & & & & \\
\hline Dibolia & $\mathrm{X}$ & $\mathrm{X}$ & $\mathrm{X}$ & $\mathrm{X}$ & & & & $\mathrm{X}$ & $\mathrm{X}$ & $\mathrm{X}$ & & \\
\hline Dimonikaea & & $\mathrm{X}$ & & & & & & & & & & \\
\hline Diphaulacosoma & & & & & $\mathrm{X}$ & & & & & & & \\
\hline Djallonia & $\mathrm{X}$ & $\mathrm{X}$ & & & & & & & & & & \\
\hline Drakensbergianella & & & & $\mathrm{X}$ & & & & & & & & \\
\hline Dunbrodya & & & & $\mathrm{X}$ & & & & & & & & \\
\hline Epitrix & $\mathrm{X}$ & $\mathrm{X}$ & $\mathrm{X}$ & $\mathrm{X}$ & $\mathrm{X}$ & & $\mathrm{X}$ & $\mathrm{X}$ & $\mathrm{X}$ & $\mathrm{X}$ & $\mathrm{X}$ & $\mathrm{X}$ \\
\hline Eriotica & & & $\mathrm{X}$ & & & & & & & & & \\
\hline Ethiopia & & & $\mathrm{X}$ & & & & & & & & & \\
\hline Eurylegna & & $\mathrm{X}$ & $\mathrm{X}$ & & & & & & & & & \\
\hline Eurylegniella & $\mathrm{X}$ & $\mathrm{X}$ & & & & & & & & & & \\
\hline Eutornus & $\mathrm{X}$ & $X$ & $\mathrm{X}$ & $\mathrm{X}$ & $\mathrm{X}$ & & & & & & & \\
\hline Gabonia & $\mathrm{X}$ & $\mathrm{X}$ & $\mathrm{X}$ & $\mathrm{X}$ & & & & & & & & \\
\hline Guilelmia & & $\mathrm{X}$ & & & & & & & & & & \\
\hline Guinerestia & $\mathrm{X}$ & $\mathrm{X}$ & & & & & & & & & & \\
\hline Halticopsis & & & $\mathrm{X}$ & & & & & & & & & \\
\hline Halticotropis & & & & & $\mathrm{X}$ & & & & & & & \\
\hline Hemipyxis & $\mathrm{X}$ & $\mathrm{X}$ & $\mathrm{X}$ & $\mathrm{X}$ & $\mathrm{X}$ & & & $\mathrm{X}$ & & & $\mathrm{X}$ & $\mathrm{X}$ \\
\hline Hildenbrandtina & & & & & $\mathrm{X}$ & & & & & & & \\
\hline Homichloda & & $\mathrm{X}$ & $\mathrm{X}$ & $\mathrm{X}$ & & & & & & & & \\
\hline Hyphasis & & & & & & & $?$ & $\mathrm{X}$ & & & $\mathrm{X}$ & \\
\hline Kanonga & & $\mathrm{X}$ & $\mathrm{X}$ & & & & & & & & & \\
\hline Kenialtica & $\mathrm{X}$ & $X$ & $X$ & $\mathrm{X}$ & $\mathrm{X}$ & & & & & & & \\
\hline Kimongona & & $\mathrm{X}$ & & & & & & & & & & \\
\hline Lampedona & & $\mathrm{X}$ & $\mathrm{X}$ & & & & & & & & & \\
\hline Lepialtica & & $\mathrm{X}$ & & & & & & & & & & \\
\hline Longitarsus & $\mathrm{X}$ & $X$ & $X$ & $\mathrm{X}$ & $X$ & $\mathrm{X}$ & $\mathrm{X}$ & $X$ & $\mathrm{X}$ & $\mathrm{X}$ & $X$ & $X$ \\
\hline Luperomorpha & & $X$ & & & & & & $X$ & & & $X$ & $X$ \\
\hline Lypnea & $\mathrm{X}$ & $\mathrm{X}$ & $\mathrm{X}$ & $\mathrm{X}$ & $\mathrm{X}$ & & & $\mathrm{X}$ & & & $\mathrm{X}$ & $\mathrm{X}$ \\
\hline Malvernia & & & $\mathrm{X}$ & $X$ & & & & & & & & \\
\hline Manobia & $\mathrm{X}$ & $\mathrm{X}$ & & & & & & $X$ & & & $\mathrm{X}$ & $\mathrm{X}$ \\
\hline Mesocrepis & & & $\mathrm{X}$ & $\mathrm{X}$ & & & & & & & & \\
\hline
\end{tabular}




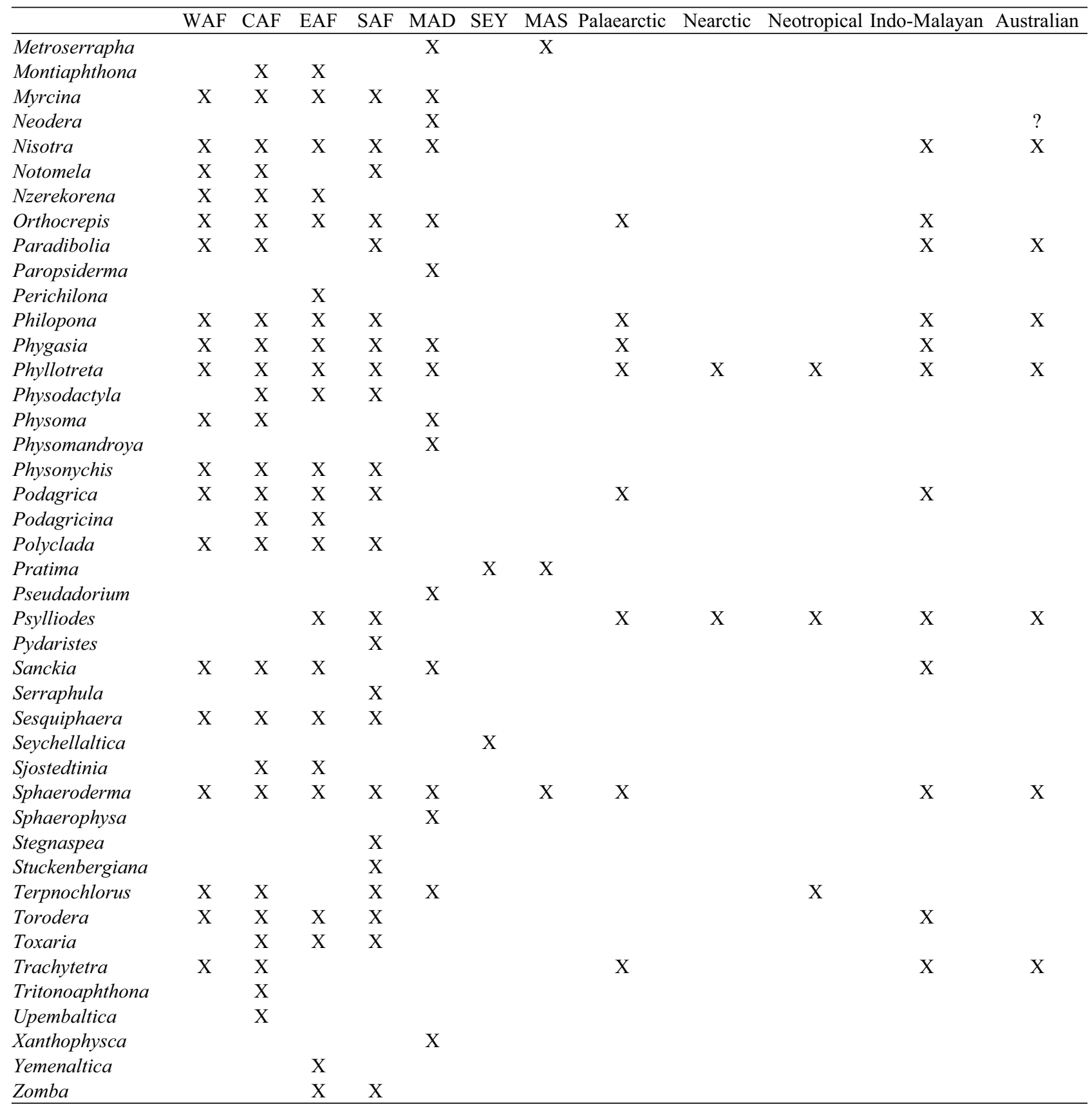

Distribution. Guinea, Sierra Leone, Ghana, Nigeria, Cameroon, and Democratic Republic of Congo; eastern Palaearctic, IndoMalayan and Australian regions.

Ecology. No information.

Notes. Five species in Sub-Saharan Africa.

\section{Tritonaphthona Bechyné, 1960}

References. Bechyné, 1960a: 70.

Type species. Aphthona longicornis Laboissière, 1942: 21 (Albert National Park), by original designation.

Distribution. Democratic Republic of Congo.

Ecology. No information.

Notes. One species.

\section{[Thrymnes Weise, 1895]}

=Gabonia Jacoby, 1893

\section{[Tropidophora Thomson, 1858]}

=Physoma Clark, 1863

\section{Upembaltica Bechyné, 1960}

References. Bechyné, 1960a: 53; Biondi \& D’Alessandro, 2003: 104.

Type species. Upembaltica scolytina Bechyné, 1960a: 53-54 (Upemba National Park: Lupiala; Kaswabilenga), by original designation.

Distribution. Democratic Republic of Congo and Gabon(!).

Ecology. No information.

Notes. One species.

[Weiseana Jacoby, 1906]

=Homichloda Weise, 1902 


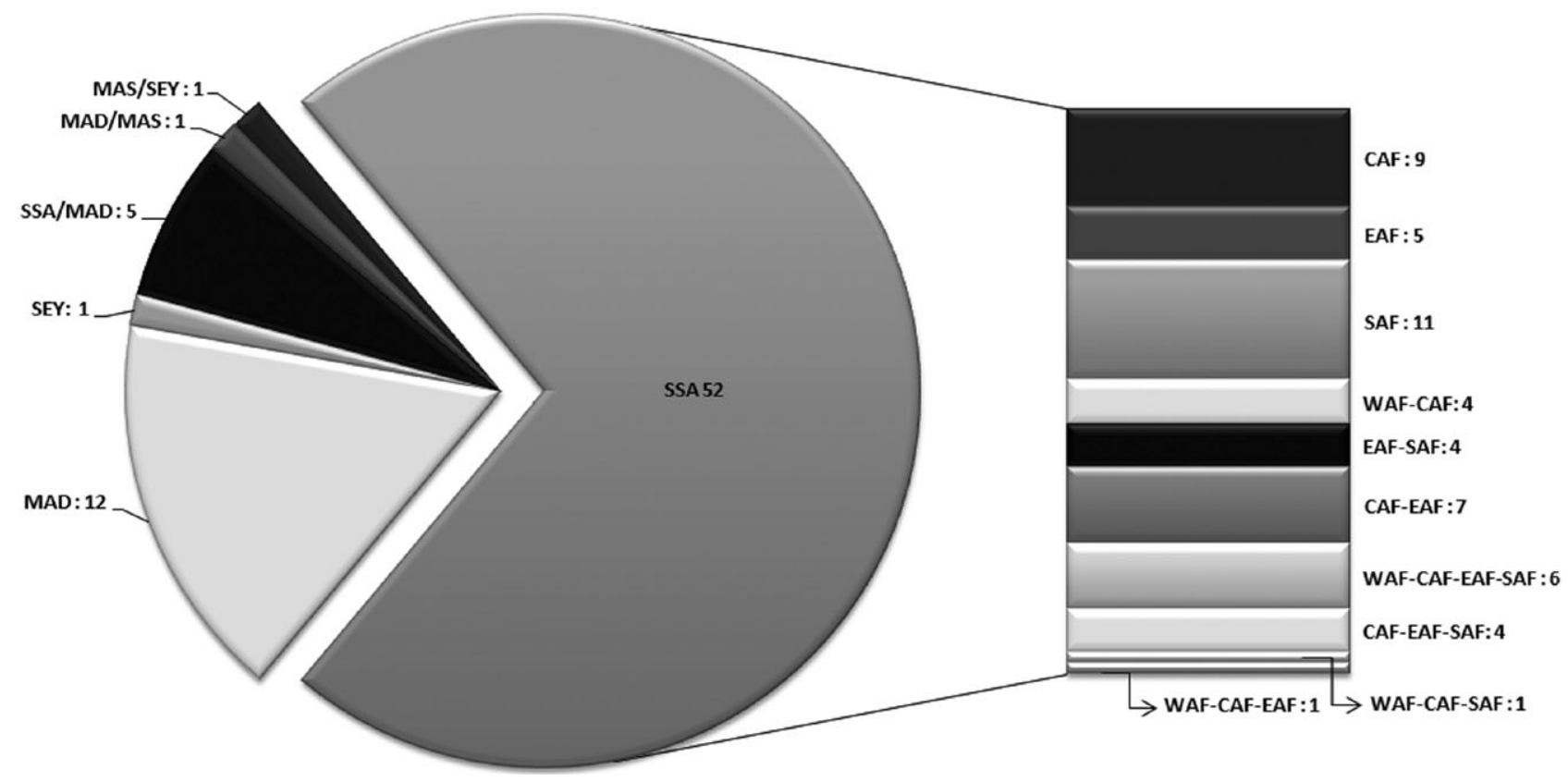

Fig. 3. Number of flea beetle genera endemic to the various areas of the Afrotropical region (see Material and methods for abbreviations).

\section{Xanthophysca Fairmaire, 1901}

References. Fairmaire, 1901: 242.

Type species. Xanthophysca perrieri Fairmaire, 1901: 242 (Madagascar: Saberbieville), by monotypy.

\section{Distribution. Madagascar.}

Ecology. No information.

Notes. One species with different colour forms.

\section{[Xenaltica Baly, 1875]}

$=$ Myrcina Chapuis, 1875

\section{Yemenaltica Scherer, 1985}

References. Scherer, 1985: 86; Medvedev, 1996: 261.

Type species. Yemenaltica scorteccii Scherer, 1985: 86 (Yemen: El Kasaba), by original designation.

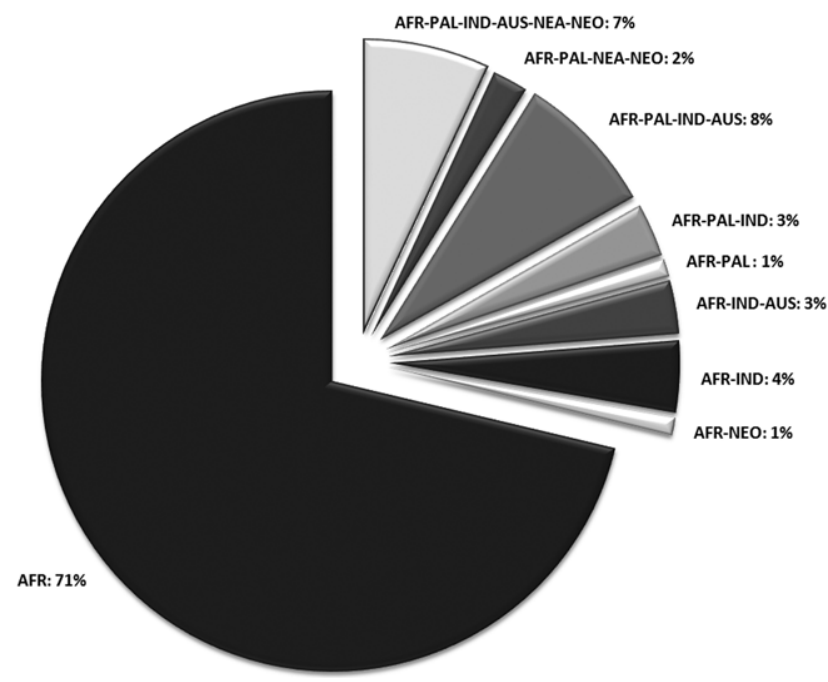

Fig. 4. Distribution of Afrotropical flea beetle genera in the different zoogeographical regions (see Material and methods for abbreviations).
Distribution. Southern Arabian Peninsula (Saudi Arabia, North Yemen and Oman).

Ecology. No information.

Notes. One species.

\section{Zomba Bryant, 1922}

References. Bryant, 1922a: 263.

Type species. Zomba gossypii Bryant, 1922a: 264 (Nyasaland: Luchenza; N.W. Rhodesia: Livingstone), by monotypy.

Distribution. Malawi and Zimbabwe.

Ecology. The only species in this genus was collected on cotton plants, Gossypium sp. (Malvaceae) (Bryant, 1922a).

Notes. One species. This genus is particularly interesting because it is the only representative of the tribe Monoplatini that occurs in the Afrotropical region. Monoplatini practically

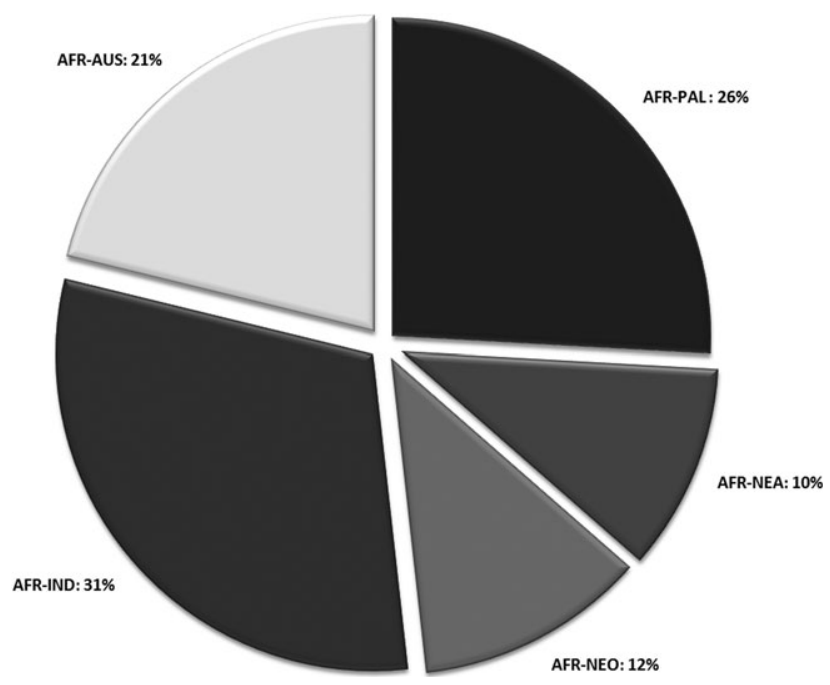

Fig. 5. Percentage of flea beetle genera co-occurring in the Afrotropical and another zoogeographical region (see Material and methods for abbreviations). 


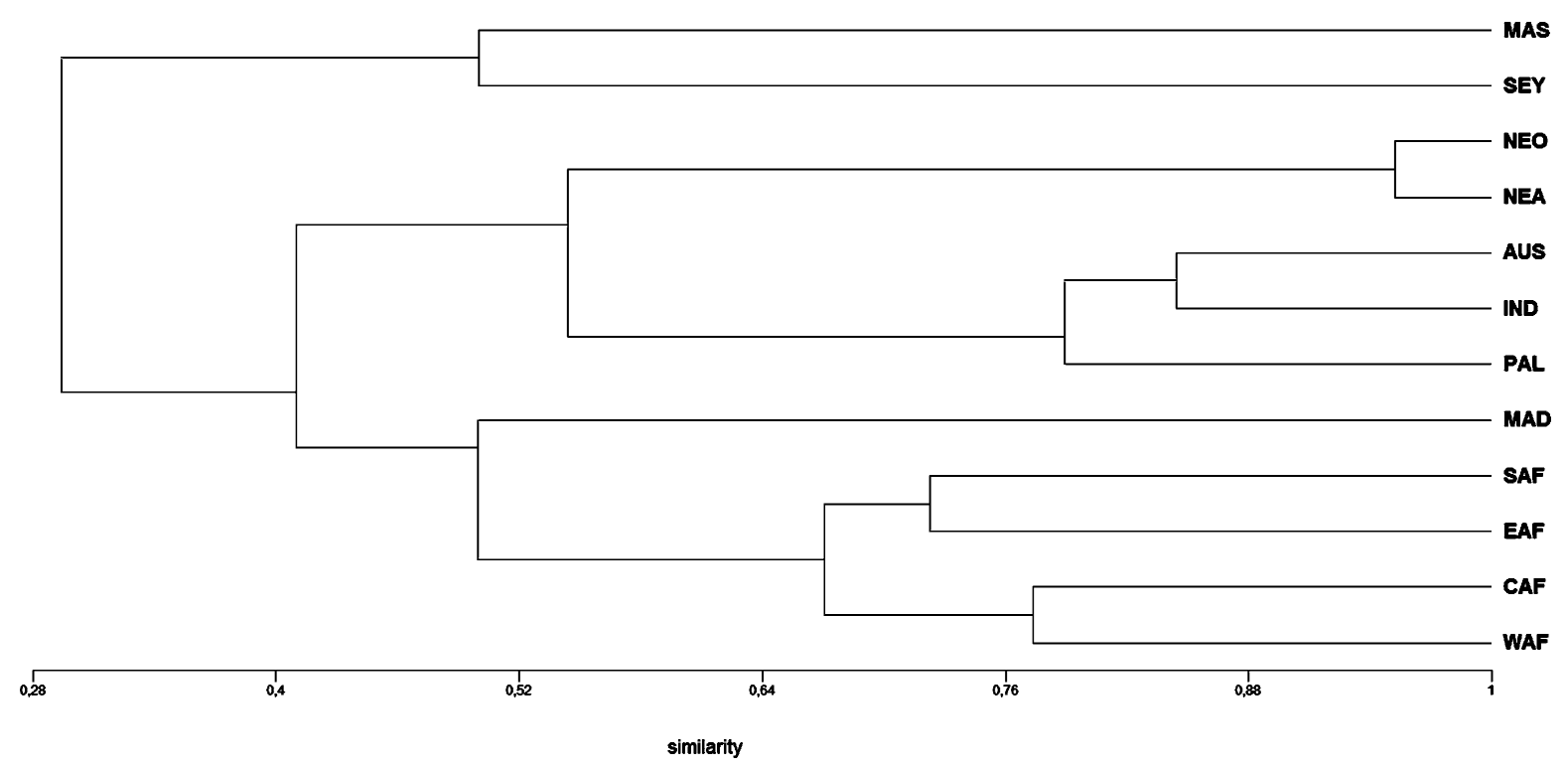

Fig. 6. Faunistic similarities of the different areas of the Afrotropical region with those of other zoogeographical regions [Coincidence index and WPGMA clustering method (Weighted Pair Group Method using Arithmetic averaging) (cf. Biondi, 2006)] (see Material and methods for abbreviations).

exclusively occur in the Neotropical and southern part of the Nearctic regions. The only exceptions are Zomba and Opisthopygme Blackburn (1896: 41); two species of the latter occur in the Australian region.

\section{DISCUSSION}

Based on this catalogue, of the 101 flea beetle genera, 84 occur in Sub-Saharan Africa (CAF: 64; EAF; 52; SAF: 52; WAF: 40), 37 in Madagascar, 8 in the Mascarene Islands and 5 in the Seychelles Islands (Table 1, Fig. 2). However, these numbers are provisional as information concerning the Afrotropical flea beetle fauna is limited, particularly for Madagascar. This preliminary overview indicates that this fauna is clearly separated from those of other zoogeographical regions (Fig. 6). The geographic distribution of Afrotropical flea beetle genera has distinct Malagasy and Sub-Saharan African components (Fig. 6).

The percentage of genera of Alticinae that are endemic to the Afrotropical region is very high $(71.0 \%)$, with the following distribution: Sub-Saharan Africa, 52 genera; Madagascar, 13; Seychelles Islands, 1; Sub-Saharan Africa-Madagascar, 4; Madagascar-Mascarene Islands, 1; Seychelles-Mascarene Islands, 1 (Fig. 3). Within the endemic Sub-Saharan African component, only 6 genera occur in all four subregions, while 25 genera occur only in one subregion (SAF: 11; CAF: 9; EAF: 5). There are no endemic flea beetle genera in WAF (Fig. 3).

The percentage of genera occurring in both the Afrotropical and another zoogeographical region is $29.0 \%$, with the cosmopolitan component significant and well represented $[7.0 \%$ of the total (101)] (Fig. 4). Of those shared genera, the Afrotropical region shares the highest percentage with the Indo-Malayan region (31.0\%) (Fig. $5)$. In this case, the presence of some genera in both regions may be due to a possible Gondwanian origin, such as Sanckia, which mainly occurs in Madagascar although species are found in the Sub-Saharan Africa and southern part of the Indo-Malayan region; Torodera, occurs in Sub-Saharan Africa and the Indo-Malayan region and is absent from Madagascar; Amphimela, Nisotra, and Paradibolia, occur in the Afrotropical, IndoMalayan and the Australian regions; and Bikasha, occurs both on the Seychelles Islands and peninsula of Vietnam. Other genera, such as Hemipyxis, Luperomorpha, Lypnea, Manobia, Philopona and Trachytetra occur not only in the Afrotropical (including Madagascar although infrequently), Indo-Malayan and Australian regions, but also in the eastern part of the Palaearctic region. $26.0 \%$ of the shared flea beetle genera occur in the Palaearctic region (Fig. 5), including the unique Pan-African flea beetle genus, Angulaphthona, which occurs in Mediterranean Africa, Sub-Saharan Africa and Madagascar (cf. Biondi \& D'Alessandro, 2006). A slightly lower percentage of genera $(21.0 \%)$ occur in both the Afrotropical and Australian regions, although all can also be found throughout the Indo-Malayan region.

As expected, a low percentage of genera occurs in both the Afrotropical and Nearctic regions (10.0\%), and in Afrotropical and Neotropical regions (12.0\%). All genera common to the Afrotropical, Nearctic and Neotropical regions are also found in all other zoogeographical regions with the exception of the genus Terpnochlorus, which only occurs in the Afrotropical region, Venezuela and Mexico (cf. Furth \& Suzuki, 1994). Moreover, the possible synonymy between the genera Abrarius from Madagascar and Gioia from South America (see above), if confirmed, could indicate an interesting zoogeographical connection among the ancient regions of the Gondwana.

Other likely Gondwanian elements in the Afrotropical flea beetle fauna are: 
1. Zomba, this unique Afrotropical genus belonging to the tribe Monoplatini which mainly occurs in the Neotropical region with a few species in the Nearctic region. The genus Opisthopygme belonging to this tribe of flea beetles is also present in Australia (see above).

2. Two new flea beetle genera to be described, preliminarily mentioned here, that occur in Madagascar and South Africa (Western Cape Province) (Biondi \& D'Alessandro, in prep.). Both those genera have clavate or sub-clavate antennae with 11 segments, and are subspherical in shape and very small, characteristics they share with related genera in Central America, such as: Bubiscus Savini, Furth \& Joly (2009: 53), a recently described Costa Rican genus (1 species); Normaltica Konstantinov (2002: 2), an endemic genus of Great Antilles (2 species); Clavicornaltica Scherer (1974: 58), a genus occurring in the Indo-Malayan (18 species) and Australian regions (1 species) (cf. Konstantinov \& Duckett, 2005). Other very closely related flea beetle genera but with a reduced number of antennal segments, are: Kiskeya Konstantinov \& Chamorro-Lacayo (2006: 276), which has nine-segmented clavate antennae, with 2 species in the Dominican Republic; and Monotalla Bechyné (1956: 588), which has ten-segmented clavate antennae, with 1 species in Guadalupe (cf. Savini \& Furth, 2001: 907).

\section{REFERENCES}

Allard E. 1889: Diagnoses de Coléoptères nouveaux. Le Naturaliste (Sér. 2) 47: 43.

BALY J.S. 1864: Descriptions of new genera and species of Phytophaga. Ann. Mag. Nat. Hist. (3rd ser.) 14: 433-442.

BALY J.S. 1875: Descriptions of new genera and species of Phytophaga. Trans. Entomol. Soc. Lond. 1875: 23-31.

BALY J.S. 1876a: Descriptions of new genera and species of Halticinae. Trans. Entomol. Soc. Lond. 1876: 433-449.

BALY J.S. 1876b: Descriptions of new genus and of new species of Halticinae. Trans. Entomol. Soc. Lond. 1876: 581-602.

BALY J.S. 1877: Descriptions of new genera of uncharacterized species of Halticinae. Trans. Entomol. Soc. Lond. 1877: 157-184; 283-323.

BALY J.S. 1878a: Characters of new genera and of some undescribed species of phytophagous beetles. Ann. Mag. Nat. Hist. (5th ser.) 1: 38-44.

BALY J.S. 1878b: Diagnoses of a new genus and some undescribed species of African Phytophaga. Entomol. Mon. Mag. 14: 204-206.

BALY J.S. 1878c: Descriptions of genera and species of Australian phytophagous beetles. J. Linn. Soc. (Zool.) 13: 458-479.

BALY J.S. 1881: Description of new species of Galerucidae. Trans. Entomol. Soc. Lond. 1881: 51-59.

BECERRA J.X. 2004: Molecular systematics of Blepharida beetles (Chrysomelidae: Alticinae) and relatives. Mol. Phylogenet. Evol. 30: 107-117.

Bechyné J. 1947a: Chrysomeloidea Madagassa (Coleoptera). Acta Entomol. Mus. Natn. Prag. 25: 29-47.

Bechyné J. 1947b: Notes sur le genre Neodera Duviv. (Col. Phytophaga, Alticidae). Bull. Mens. Soc. Linn. Lyon 16: 139-140.

BeChyné J. 1948a: Novi Halticidi z Madagascaru. De novis speciebus Halticidarum Madagascariensium (Col. Phytophaga). Acta Soc. Entomol. Cechoslov. 45: 3-11.
Bechyné J. 1948b: Nouveaux Galerucides de Madagascar (Col. Phytophaga). Bull. Mens. Soc. Linn. Lyon 17: 99-101.

BECHYNÉ J. 1950: Tableau analytique des Lactica de Madagascar (Col. Phytophaga, Alticidae). Bull. Mens. Soc. Linn. Lyon 19: 220-224.

Bechyné J. 1952: Nouveaux Alticides de Madagascar. Bull. Mens. Soc. Linn. Lyon 21: 248-251.

BeCHYNÉ J. 1954a: Notes sur les Chrysomeloidea de Madagascar (Col. Phytophaga). Rev. Fr. Entomol. 21: 41-47.

Bechyné J. 1954b: Reise des Herrn G. Frey in Süd-Afrika: Alticinae (Col. Phytoph.). Entomol. Arb. Mus. G. Frey 5: 675-685.

BeChyné J. 1955a: Contributions à l'étude de la faune entomologique du Ruanda Urundi (Mission P. Basilewsky, 1953) (pars Alticinae). Ann. Mus. R. Congo Belge (Zool.) 40: 204-230.

Bechyné J. 1955b: Über die Westafrikanischen Alticiden (Col. Phytophaga). Entomol. Arb. Mus. G. Frey 6: 486-568.

BechynÉ J. 1955c: Description de deux nouveaux Alticides de Madagascar (Col. Phytophaga). Naturaliste Malgache 7: 61-62.

Bechyné J. 1955d: Reise des Herr G. Frey in Südamerika Alticidae (Col. Phyt.). Entomol. Arb. Mus. G. Frey 6: 74-266.

Bechyné J. 1956: Über die Alticiden-Sammlung Heikertinger (Col. Phytophaga). Entomol. Arb. Mus. G. Frey 7: 577-598.

Bechyné J. 1957: Die von Dr. Christa Lindemann und Nina Pavlitzki in Tanganjika gesammelten Alticiden (Col. Phytophaga). Veröff. Zool. Stsamml. Münch. 4: 151-182.

BeChyné J. 1958a: Contribution à l'étude des Chrysomeloidea des Iles Mascareignes. II. Alticidae. Bull. Maurit. Inst. 5: 83-93.

BeChyNÉ J. 1958b: Notes sur quelques Oedionychini de Madagascar. Bull. Mens. Soc. Linn. Lyon 28: 318-323.

BeChyNé J. 1958c: Notes sur les Alticides de Madagascar (Col. Phytophaga). Bull. Mens. Soc. Linn. Lyon 27: 8-11.

BeChYNÉ J. 1959a: Observations sur les Alticides recueillis au Congo Belge par M.A. Collart (Coleoptera, Phytophaga). Bull. Inst. R. Sci. Nat. Belg. 35: 1-36.

BeChyNÉ J. 1959b: Coleoptera: Chrysomelidae II. S. Afr. Anim. Life 6: 227-238.

Bechyné J. 1959c: Notes sur quelques Oedionychini de Madagascar. Bull. Mens. Soc. Linn. Lyon 28: 318-323.

BECHYNÉ J. 1959d: Tableau analytique du genre Nisotra Baly de Madagascar (Col. Phytophaga, Alticidae). Bull. Mens. Soc. Linn. Lyon 28: 153-157.

BeChYNÉ J. 1960a: Notes sur les Alticides Africains des collections de l'Institut Royal des Sciences Naturelles de Belgique (Coleoptera, Phytophaga). Bull. Inst. R. Sci. Nat. Belg. 36: $1-32$.

Bechyné J. 1960b: Alticidae (Coleoptera, Phytophaga). Exploration du Parc National de l'Upemba. Mission G.F. de Witte (1946-1949). Institut Parcs Nationaux du Congo Belge, Bruxelles, fasc. no. 59, pp. 39-114.

BeChYNÉ J. 1964: Notizen zu den madagassischen Chrysomeloidea (Col. Phytophaga). Mitt. Münch. Entomol. Ges. 54: 68-161.

Bechyné J. 1968: Contribution à la faune du Congo (Brazzaville). Mission A. Villiers et A. Descarpentries. LXXXI. Coleoptères Alticidae. Bull. Inst. Fr. Afr. Noire (A) 30: $1687-1728$.

Bechyné J. \& Springlova de Bechyné B. 1975: Notas sobre la serie filetica de Monomacra y sus formas convergentes (Col. Phytophaga, Alticidae). Revta Fac. Agron. Univ. Cent. Venez. 8: $25-140$.

Bedel L. 1898: Faune des coleoptères du bassin de la Seine. Ann. Soc. Entomol. Fr. 5: 181-228. 
Berthold A.A. 1827: Latreille's Naturliche Familien des Thierreichs. Aus dem Französischen. Mit Ammerkungen und Zusätzen. Landes-Industrie-Comptoir, Weimar, 606 pp.

Biondi M. 1994: Contributo alla conoscenza dei Chrysomelidae Alticinae (Coleoptera) della Sierra Leone. 1a. Nota. Quad. Accad. Naz. Lincei 267: 423-438.

BIONDI M. 1996: The genus Psylliodes in the Afrotropical Region with description of four new species from Kenya, Tanzania and South Africa (Coleoptera, Chrysomelidae) Fragm. Entomol. 28: 257-276.

Biondi M. 1998a: The genus Malvernia Jacoby with description of a new species from South Africa (Coleoptera, Chrysomelidae). Nouv. Revue Entomol. (N.S.) 15: 37-43.

Biondi M. 1998b: Revision of the Genus Chirodica Germar with description of five new species (Coleoptera: Chrysomelidae) In Biondi M., Daccordi M. \& Furth D.G. (eds): Proceedings of the Fourth International Symposium on the Chrysomelidae, Firenze 1996. Museo Regionale di Scienze Naturali, Torino, pp. 17-48.

BIONDI M. 2000: Biodontocnema brunnea n. gen. and n. sp. from South Africa (Coleoptera: Chrysomelidae: Alticinae). Coleopt. Bull. 54: 347-350.

Biondi M. 2001a: New flea beetle genus and species (Coleoptera: Chrysomelidae, Alticinae) from Central Africa. Can. Entomol. 133: 643-649.

Biondi M. 2001b: Revision of the Chaetocnema species from Madagascar (Coleoptera, Chrysomelidae, Alticinae). Eur. J. Entomol. 98: 233-248

Biondi M. 2002a: Checklist of the Afrotropical species of the genus Chaetocnema Stephens (Coleoptera, Chrysomelidae: Alticinae): synonymies and geographical distributions. Afr. Entomol. 10: 265-284

Biondi M. 2002b: Comparative analysis of Chaetocnema Stephens and its kindred genera, with description of a new genus from the Indian Ocean (Seychelles) (Coleoptera, Chrysomelidae, Alticinae). Ital. J. Zool. 69: 22-33.

Biondi M. 2006: Il calcolo della somiglianza con dati binari nelle analisi biogeografiche. Biogeographia 37: 227-251.

Biondi M. \& D'AlessandRo P. 2003: Drakensbergianella rudebecki, new genus and new species from the mountains of the Southern Africa, and taxonomic observations on Gabonia Jacoby and related genera (Coleoptera: Chrysomelidae, Alticinae). Zool. Anz. 242: 97-106.

Biondi M. \& D'Alessandro P. 2004: Revision of the Afrotropical flea beetle genus Collartaltica Bechyné with description of two new species (Coleoptera, Chrysomelidae, Alticinae). Insect Syst. Evol. 35: 1-14.

Biondi M. \& D'AlessANDRo P. 2006: Biogeographical analysis of the flea beetle genus Chaetocnema in the Afrotropical Region: distribution patterns and areas of endemism. J. Biogeogr. 33: 720-730.

Biondi M. \& D'Alessandro P. 2007: Afroaltica subaptera, new genus and new species of flea beetles from the southern Africa (Coleoptera: Chrysomelidae: Alticinae). Eur. J. Entomol. 104: 99-103.

Biondi M. \& D'Alessandro P. 2008a: Revision of the Chaetocnema pulla species-group from the Afrotropical region with description of a new species from Central Africa (Coleoptera, Chrysomelidae). In Jolivet P., Santiago-Blay J. \& Schmitt M. (eds): Research on Chrysomelidae. Vol. 1. Brill, Leiden, Boston, pp. 265-285.

Biondi M. \& D'Alessandro P. 2008b: Taxonomical revision of the Longitarsus capensis species-group: An example of Mediterranean-southern African disjunct distributions (Coleoptera: Chrysomelidae). Eur. J. Entomol. 105: 719-736.
Biond M. \& D'Alessandro P. 2010: Revision of the Afrotropical flea beetle genus Serraphula Jacoby and description of Bechynella, a new genus from Western and Central Africa (Coleoptera, Chrysomelidae, Alticinae). Zootaxa 2444: 1-44.

BlACKBURN T. 1896: Further notes on Australian Coleoptera, with descriptions of new genera and species. Trans. R. Soc. S. Aust. 19: 35-109.

Boheman C.H. 1859: Coleoptera. Species novas descripsit. In Kongliga Svenska Fregatten Eugenies Resa omkring Jorden. Zoologi 1, Insecta. Vetenskapliga Iakttagelser, Stockholm, pp. 113-218.

BRANCSIK K. 1891: Beiträge zur Kenntnis Nossibé's und dessen Fauna nach Sendungen and Mitteilungen des Herrn P. Frey. Jh. Naturw. Ver. Trencsiner Com. 14: 123-167.

Broun T. 1880: Manual of the New Zealand Coleoptera. Part I. Office of James Hughes, Lambton Quay, Wellington, 651 pp.

BRYANT G.E. 1922a: Some new injurious Phytophaga from Africa. Bull. Entomol. Res. 12: 473-475.

BRYANT G.E. 1922b: New injurious Phytophaga from India and Brazil. Bull. Entomol. Res. 13: 261-255.

BRYANT G.E. 1927: New Halticidae (Col.) from Africa and Haiti. Ann. Mag. Nat. Hist. (9th ser.) 19: 615-622.

BRyant G.E. 1936: Some new injurious Phytophaga from British East Africa (Col.). Proc. R. Entomol. Soc. Lond. (B) 5: 217-219.

BRYANT G.E. 1943: New species of African Sphaeroderma (Halticinae, Col.). Ann. Mag. Nat. Hist. (11th ser.) 10: 486-494.

BRYANT G.E. 1944a: New species of Blepharida from Africa (Halticinae, Col.). Ann. Mag. Nat. Hist. (11th ser.) 12: 129-137.

BRYant G.E. 1944b: Two injurious species of Phytophaga (Halticinae) from Ivory Coast. Bull. Entomol. Res. 35: 141-142.

Chaboo C.S., Grobbelaar E. \& Larsen A. 2007: Fecal ecology in Leaf Beetles: Novel records in the African arrow-poison beetles, Diamphidia Gerstaecker and Polyclada Chevrolat (Chrysomelidae: Galerucinae). Coleopt. Bull. 61: 297-309.

Chapuis F. 1875: In Lacordaire T. (ed.): Histoire Naturelle des Insectes. Genera des Coléoptères. Vol. 11, Famille des Phytophages. Libraire Encyclopédique de Roret, Paris, 420 pp., 11 pls.

Chapuis F. 1879: Phytophages abyssiniens du musée civique d'histoire naturelle de Génes. Ann. Mus. Civ. Stor. Nat. Genova 15: 5-31.

Chevrolat L.A.A. 1835: In Dejean P.F.M.A (ed.): Catalogue des Coléoptères de la Collection de M. le Comte Dejean. 2nd $e d$. Librairie Méquignon-Marvis Père et Fils, Paris, pp. 257-360.

Chevrolat L.A.A. 1837: In Dejean P.F.M.A (ed.): Catalogue des Coléoptères de la Collection de M. le Comte Dejean. 3rd ed., revue, corrigée et augmentée, livr. 5. Librairie Méquignon-Marvis Père et Fils, Paris, pp. 385-503.

Chevrolat L.A.A. 1842: In d'Orbigny C.D. (ed.): Dictionnaire Universel d'Histoire Naturelle, Vol. 2. C. Renard, Paris, pp. $1-795$.

Chevrolat L.A.A. 1843: In d'Orbigny C.D. (ed.): Dictionnaire Universel d'Histoire Naturelle, Vol. 3. C. Renard, Paris, pp. $1-744$.

Chevrolat L.A.A. 1845: In d'Orbigny C.D. (ed.): Dictionnaire Universel d'Histoire Naturelle, Vol. 6. C. Renard, Paris, pp. $1-792$.

CHÛJô M. 1936: Studies on the Chrysomelidae in the Japanese Empire (VIII), subfamily Halticinae (4-6). Trans. Nat. Hist. Soc. Formosa 148: 15-30, 84-92, 108-114.

CHÛJô M. 1937: Studies on the Chrysomelidae in the Japanese Empire (VIII), subfamily Halticinae (7-10). Trans. Nat. Hist. Soc. Formosa 161: 35-48; 162: 52-58. 
Clark H. 1860: Catalogue of Halticidae in the Collection of the British Museum. Physapodes and Oedipodes. Part I. Order of Trustees, London, $301 \mathrm{pp}$., $10 \mathrm{pls}$.

CLARK H. 1863: Catalogue of Halticidae; being a continuation of the British Museum Catalogue, Part 1, 1860. J. Entomol. 9: 163-174.

Cox M.L. 1997: Homichloda barkeri (Jacoby) (Coleoptera: Chrysomelidae: Alticinae), a candidate agent for the biocontrol of prickly acacia, Acacia nilotica (Mimosaceae) in Australia. J. Nat. Hist. 31: 935-964.

CURTIS J. 1833: British Entomology; being Illustrations and Descriptions of the Genera of Insects found in Great Britain and Ireland. Vol. 10. London, pls 434-481.

D'Alessandro P. \& Biondi M. in press: The Afrotropical genus Afroaltica Biondi \& D'Alessandro (Coleoptera, Chrysomelidae, Alticinae): new data and description of a second new species from Limpopo (Republic of South Africa). Ann. Soc. Entomol. Fr.

DöBERL M. 2008: Über die Typen einiger afrikanischer Arten der Gattung Altica Geoffroy, 1762 (Coleoptera: Chrysomelidae: Alticinae). Acta Coleopt. 24: 35-39.

DöBERL M. 2010: Beitrag zur Kenntnis der afrotropischen Arten von Altica Geoffroy, 1762 unter Ausschluss der Arten Madagaskars (Coleoptera: Chrysomelidae, Alticinae). Entomol. Z. 120: $51-72$.

Doguet S. 1974: Contribution à l'étude des Alticinae des Iles Mascareignes (Col. Chrysomelidae). Nouv. Rev. Entomol. 4: $119-126$.

Doguet S. 1979: Insects of Saudi Arabia. Coleoptera: Fam. Chrysomelidae, Subfam. Halticinae. Fauna of Saudi Arabia 1: 308-316.

DRINKWATER T.W. 1989: Buphonella murina Gerstaecker (Coleoptera: Chrysomelidae) another Maize rootworm in South Africa. Phytophylactica 21: 315-316.

Duckett C.N., GillesPie J.J. \& KJeR K.M. 2004: Relationships among the subfamilies of Chrysomelidae inferred from small subunit ribosomal DNA and morphology, with special emphasis on the relationship among the flea beetles and the Galerucinae. In Jolivet P.H., Santiago-Blay J.A. \& Schmit M. (eds): New Contributions to the Biology of Chrysomelidae. SPB Academic Publishers, The Hague, pp. 3-18.

Duvivier A. 1891: Diagnoses de phytophages madégasses. Ann. Soc. Entomol. Belg. 35: 238-245; 313-321; 423-424.

Duvivier A. 1892: Les phytophages du Chota-Nagpore. 2e Note. Ann. Soc. Entomol. Belg. 36: 396-449.

ERICHSON W.F. 1834: Coleoptera. Beiträge zur Zoologie, gesammelt auf einer Reise um die Erde, von Dr. F.J.F. Meyen. Nova Acta Acad. Caesar. Leop. Carol. 16 (Suppl.): 219-284.

ERICHSON W.F. 1847: Conspectus insectorum coleopterorum, quae in Republica Peruana observata sunt. Arch. Naturgesch. 13: $67-185$.

FABricius J.C. 1775: Systema entomologiae, sistens insectorum classes, ordines, genera, species, adiectis synonymis, locis, descriptionibus, observationibus. Officina Libraria Kortii, Flensburgi et Lipsiae, $\mathrm{xxx}+832 \mathrm{pp}$.

FABRICIUS J.C. 1787: Mantissa insectorum sistens eorum species nuper detectas adiectis characteribus genericis differentiis specificis, emendationibus, observationibus. Tom. I. Impensis C.G. Proft, Hafniae, $x x+348$ pp.

FABricius J.C. 1798: Supplementum entomologiae systematicae. Proft et Storch, Hafniae, iv +572 pp.

Fairmaire L. 1883a: Diagnoses de Coléoptères Abyssins. Le Naturaliste (sér. 2) 25: 197.

FAIRMAIRE L. 1883b: Descriptions de Coléoptères nouveaux ou peu connus récoltés par Mr. Raffray en Abyssinie. Ann. Soc. Entomol. Fr. 3: 89-112.
FAIRMAiRe L. 1884: Note sur les Coléoptères recueillis par M. Ach. Raffray à Madagascar et descriptions des espèces nouvelles. 1-ère partie. Ann. Soc. Entomol. Fr. 4: 225-242.

FAIRMAIRE L. 1886: Notes sur les Coléoptères recueillis par M. Raffray a Madagascar et descriptions des espèces nouvelles. Ann. Soc. Entomol. Fr. 6: 31-96.

Fairmaire L. 1888: Notes sur les Coléoptères des environs de Pekin. Rev. Fr. Entomol. 7: 111-160.

FAIRMAIRE L. 1898: Materiaux pour la faune coléoptèrique de la région malgache. $5^{\circ}$ note. Ann. Soc. Entomol. Belg. 42: 390-439.

FAIRMAIRE L. 1901: Materiaux pour la faune coléoptèrique de la région malgache ( $11^{\circ}$ note). Rev. Fr. Entomol. 20: 101-248.

FAIRMAIRE L. 1902: Materiaux pour la faune coleopterique malgache. $13^{\circ}$ note. Ann. Soc. Entomol. Belg. 46: 236-271.

FAIRMAIRE L. 1904: Materiaux pour la faune coléoptèrique malgache $18^{\circ}$ note. Ann. Soc. Entomol. Belg. 48: 225-276.

Ferreira M.C. 1963: Catalogo dos Coleopteros de Moçambique: Chrysomelidae Alticinae. Revta Entomol. Moçamb. 6: 515-520.

FISCHER VON WALDHEIM G. 1824: Entomographia imperii russici; genera insectorum systematica exposita et analysi iconographica instructa, vol. 2. Société Impériale des Naturalistes, Mosquae, $262 \mathrm{pp}$.

Forster J.R. 1771: Novae Species Insectorum. Centuria I. Davies and White, London, $100 \mathrm{pp}$.

Foudras A.C.M.E. 1860: Altisides. Ann. Soc. Linn. Lyon 6: 137-384.

FuRTh D.G. 1985: Some flea beetles and their foodplants from Kenya (Chrysomelidae: Alticinae). Coleopt. Bull. 39: 259-263.

FuRTH D.G. 1998: New world Blepharida Chevrolat 1836 (Coleoptera: Chrysomelidae: Alticinae). Mem. Entomol. Soc. Wash. 21: 3-109.

FurTh D.G. \& LeE J.E. 2000: Similarity of the Blepharida-group genera using larval and adult characters (Coleoptera: Chrysomelidae: Alticinae). J. N. Y. Entomol. Soc. 108: 26-51.

FurTh D.G. \& SuZUKI K. 1994: Character correlation studies of problematic genera of Alticinae in relation to Galerucinae (Coleoptera: Chrysomelidae). In Furth D.G. (ed.): Proceedings of the Third International Symposium on the Chrysomelidae, Beijing 1992. Backhuys, Leiden, pp. 116-135.

FurTh D.G. \& SuzuKi K. 1998: Studies of Oriental and Australian Alticinae genera based on the comparative morphology of the metafemoral spring, genitalia, and hind wing venation. In Biondi M., Daccordi M. \& Furth D.G. (eds): Proceedings of the Fourth International Symposium on the Chrysomelidae, Firenze 1996. Museo Regionale di Scienze Naturali, Torino, pp. 91-124.

FuRTh D.G. \& Young D.A. 1988: Relationships of herbivore feeding and plant flavonoids (Coleoptera: Chrysomelidae and Anacardiaceae: Rhus). Oecologia 74: 496-500.

Geoffroy E.L. 1785: In Fourcroy A.F. de (ed.): Entomologia Parisiensis, sive catalogus Insectorum, quae in agro parisiensi reperiuntus - secundum methodam Geoffraeriam in sectiones, genera et species distributii; cui addita sunt nomina trivialia et fere recentae novae species. Pars prima. Paris, $544 \mathrm{pp}$.

Germar E.F. 1834: Description du genre Chirodica. Rev. Entomol. 2: 1-2.

GERSTAECKER C.E.A. 1871: Beiträge zur Insektenfauna von Zanzibar. III. Coleoptera. Arch. Naturgesch. 37: 42-86.

Gerstaecker C.E.A. 1855: Diagnosen der von Peters in Mossambique gesammelten Kafer u. Hymenoptera. Monatsber. Akad. Wissensch. Berlin 1855: 636-639. 
Gómez-Zurita J., Hunt T., Kopliku F. \& Vogler A.P. 2007: Recalibrated tree of leaf beetles (Chrysomelidae) indicates independent diversification of Angiosperms and their insect herbivores. PLoS ONE 2(4): e360. doi: 10.1371/journal.pone. 0000360

Gould J. 1842: [Meeting of February 8, 1842]. Mr. Gould pointed out the generic characters of two new genera of Finches. Proc. Zool. Soc. Lond. 10: 17-18.

Graf D.L. \& Cummings K.S. 2007: Review of the systematics and global diversity of freshwater mussel species (Bivalvia: Unionoida). J. Mollusc. Stud. 73: 291-314.

HAROLd von E. 1875: Pydaristes, eine neue Gattung der Halticidae. Stett. Entomol. Ztg 36: 446-448.

Harold von E. 1877a: Coleopterorum species novae. Mitt. Münch. Entomol. Ver. 1: 97-111.

HAROLD vON E. 1877b: Beitrage zur Kaferfauna von Japan (zweites stuck). Berl. Entomol. Z. 21: 433-434.

Harold von E. 1878: Synonymies. Petites Nouv. Entomol. 2: 206.

Heyden von L. 1887: Kleine coleopterologische Mitteilungen. Wien. Entomol. Ztg 6: 98.

Heikertinger F. 1909: Beschreibung zweir neuer Halticinen. Verh. K. K. Zool. Bot. Ges. Wien 59: 361-374.

Heikertinger F. 1925: Monographie der Halticinengattung Derocrepis Weise (Coleopt., Chrysomelidae). Wien. Entomol. Ztg 42: 95-178.

Heikertinger F. \& Csiki C. 1940: Coleopterorum Catalogus auspiciis et auxilio W. Junk editus a S. Schenkling. Partes 160 et 169. Chrysomelidae: Halticinae, vol. 25. Dr. W. Junk, Gravenhage, pp. 337-635.

Hope F.W. 1840: The Coleopterist's Manual, Part the Third, Containing Various Families, Genera, and Species, of Beetles, Recorded by Linneus and Fabricius. Also, Descriptions of Newly Discovered and Unpublished Insects. Bowdery \& Kerby, London, $191 \mathrm{pp}$.

ILLIGER J.C.W. 1801: Namen der Insekten-Gattungen, ihr Genitiv, ihr Grammatisches Geschlecht, ihr Silbenmass, ihre Herleitung; zugleich mit den deutschen Benennungen. Mag. Insekten 1: 125-155.

International Commission on Zoological Nomenclature 1999: International Code of Zoological Nomenclature. 4th ed. International Trust for Zoological Nomenclature, The Natural History Museum, London, xxix $+306 \mathrm{pp}$.

JACOBSON G.G. 1925: Chrysomelidae (Coleoptera) palaearctici novi vel parum cogniti. V-VI. Ann. Mus. Zool. Leningrad 26: 231-276.

JACOBY M. 1885: Descriptions of new genera and species of phytophagous Coleoptera from the Indo-Malayan and AustroMalayan subregions, contained in the Genoa Civic Museum. Ann. Mus. Civ. Stor. Nat. Genova (ser. 2) 2: 20-76.

JACOBY M. 1888: Descriptions of new or little-known species of phytophagous Coleoptera from Africa and Madagascar. Trans. Entomol. Soc. Lond. 1888: 189-206.

JACOBY M. 1889: List of the phytophagous Coleoptera obtained by Signor L. Fea at Burmah and Tenasserim, with descriptions of new species. Ann. Mus. Civ. Stor. Nat. Genova (ser. 2) 7: $147-237$

JACOBY M. 1892a: Descriptions of some new genera and new species of phytophagous Coleoptera from Madagascar. Proc. R. Entomol. Soc. Lond. 1892: 564-579.

JACOBY M. 1892b: Viaggio di Leonardo Fea in Birmania e regioni vicine. LI. Description of the new genera and species of the phytophagous Coleoptera. Ann. Mus. Civ. Stor. Nat. Genova (ser. 2) 12: 869-999.
JACOBY M. 1893: Descriptions of some new species of Eumolpidae and Halticidae from Africa (Gaboon). Entomologist (Suppl.) 26: 97-102.

JACOBY M. 1895: Contributions to the knowledge of African phytophagous Coleoptera. Part II. Trans. Entomol. Soc. Lond. 1895: 317-341.

JACOBY M. 1897: Further contributions to the knowledge of the phytophagous Coleoptera of Africa, including Madagascar. Proc. R. Entomol. Soc. Lond. 1897: 527-577.

JACOBY M. 1899a: Some new genera and species of phytophagous Coleoptera collected during Captain Bottego's last expedition. Ann. Mus. Civ. Stor. Nat. Genova (ser. 2) 19: 521-535.

JACOBY M. 1899b: Additions to the knowledge of the phytophagous Coleoptera of Africa. Part II. Proc. R. Entomol. Soc. Lond. 1899: 339-380.

JACOBY M. 1900: On new genera and species of phytophagous Coleoptera from South and Central Africa. Proc. R. Entomol. Soc. Lond. 1900: 203-266.

JACOBY M. 1901: Descriptions of some new genera and species of phytophagous Coleoptera from Madagascar. Ann. Soc. Entomol. Belg. 45: 287-303.

JACOBY M. 1903a: A further contribution to our knowledge of African phytophagous Coleoptera. Part II. Trans. Entomol. Soc. Lond. 1903: 1-38.

JACOBY M. 1903b: Descriptions of the new genera and species of phytophagous Coleoptera obtained by Mr. H.L. Andrewes and Mr. T.R.D. Bell at the Nilgiri Hills and Kanara. Ann. Soc. Entomol. Belg. 67: 80-128.

JACOBY M. 1906: Descriptions of new genera and species of African Halticinae and Galerucinae. Trans. Entomol. Soc. Lond. 1906: 11-52.

JACOBY M. 1907: Voyage de M. Maurice de Rothschild en Ethiopie et dans l'Afrique orientale (1904-1906). Espèces nouvelles de Chrysomelidae (Col.). Ann. Soc. Entomol. Fr. 76: $515-525$.

Jolivet P. \& Hawkeswood T.J. 1995: Host-Plants of Chrysomelidae of the World. An Essay about the Relationships between the Leaf-Beetles and their Food-Plants. Backhuys, Leiden, $281 \mathrm{pp}$.

Jolivet P. \& Verma K.K. 2002: Biology of Leaf Beetles. Intercept, Andover, Hampshire, xiv +332 pp.

KIESENWETTER VON H. 1877: In Erichson W.F. (ed.): Naturgeschichte der Insecten Deutschlands. Erste Abtheilung. Coleoptera. Fünfter Band, Erste Lieferung, Bogen 1-12. Nicolaische Verlags-Buchhandlung, Berlin, pp. 1-887.

Koch J.D.W. 1803: Monographie der Floehkaefer. Entomol. Hefte 2(1): 3-90.

Kolbe H.J. 1894: Die Pfeilgiftkafer der Kalahari-Wuste, Diamphidia simplex Pering. (locusta Fairm.). Stettin. Entomol. Ztg 55: 79-86.

Konstantinov A.S. 1998: Revision of the Palearctic species of Aphthona Chevrolat and cladistic classification of the Aphthonini (Coleoptera: Chrysomelidae: Alticinae). Mem. Entomol. 11: 1-429.

Konstantinov A.S. 2002: A new genus of flea beetles from Greater Antilles (Coleoptera: Chrysomelidae). Zootaxa 124: $1-24$.

Konstantinov A.S. \& Chamorro-Lacayo M.L. 2006: A new genus of moss-inhabiting flea beetles (Coleoptera: Chrysomelidae) from the Dominican Republic. Coleopt. Bull. 60: 275-290.

Konstantinov A.S. \& DucketT C.N. 2005: New species of Clavicornaltica Scherer (Coleoptera: Chrysomelidae) from continental Asia. Zootaxa 1037: 49-64. 
Konstantinov A.S. \& Prathapan K.D. 2008: New generic synonyms in the Oriental flea beetles (Coleoptera: Chrysomelidae). Coleopt. Bull. 62: 381-418.

Konstantinov A.S. \& VANDERBERG N.J. 1996: Handbook of Palearctic Flea Beetles (Coleoptera: Chrysomelidae: Alticinae). Contrib. Entomol. 1: 237-439

LABOISSIÈre V. 1922: Étude des Galerucini de la collection du musée du Congo belge. Troisième Partie. Rev. Zool. Afr. 10: 233-271.

LABOISSIÈre V. 1932: Synopsis des Genres de Galerucini de Madagascar. Société Entomologique de France, Livre du Centenaire. Paris, pp. 575-592.

LABOISSIÈre V. 1933: Descriptions de trois nouveaux Galerucini du Tonkin. Bull. Mus. Natn. Hist. Nat. (A) 5: 203-208.

LABOISSIÈRE V. 1937: Nouveaux Galerucinae palearctiques. Bull. Soc. Entomol. Fr. 42: 27-31.

LABOISSIÈre V. 1941: Nouveaux Halticinae et Galerucinae de la collection du musée du Congo. Rev. Zool. Bot. Afr. 34: 316-321.

LABOISSIĖre V. 1942: Exploration du Parc National Albert. Mission G.F. De Witte (1933-1935). Halticinae (Coleoptera: Phytophaga). Fam. Chrysomelidae. Institut Parcs Nationaux du Congo Belge, Bruxelles, fasc. no. 39: pp. 3-130.

Latreille P.A. 1810: Considérations Générales sur l'Ordre Naturel des Animaux Composant les Classes des Crustacés, des Arachnides, et des Insectes. Avec un Tableau Méthodique de leurs Genres, Disposés en Familles. F. Schoell, Paris, 444 pp.

Latreille P.A. 1829: Les Crustacés, les Arachnides et les Insectes Distribués en Familles Naturelles, Ouvrage Formant les Tomes 4 et 5 de celui de M. Le Baron Cuvier sur le Règne Animale ( $2^{e} e ́ d$.), Vol. 1. Déterville, Paris, $584 \mathrm{pp}$.

Lebert H. 1879: Hydrachnides du Léman. In Forel F.A. (ed.) Fauna profonde du Lac Léman. Bull. Soc. Vaud. Sci. Nat. 16: $327-377$.

Linnaeus C. 1758: Systema Naturae per regna tria naturae: secundum classes, ordines, genera, species cum caracteribus, differentiis, synonymis, locis. Tomus I. Editio Decima, reformata. Laurentii Salvii, Stockholmiae, 824 pp.

Linnaeus C. 1761: Fauna Svecica sistens Animalia Sveciae regni: Mammalia, Aves, Amphibia, Pisces, Insecta, Vermes. Distributa per classes \& ordines, genera \& species, cum differentiis specierum, synonymis auctorum, nominibus incolarum, locis natalium, descriptionibus insectorum. Laurentii Salvii, Stockholmiae, $578 \mathrm{pp}$.

LuCAS P.H. 1849: Histoire naturelle des animaux articulés. Deuxième partie. Insectes. In: Exploration Scientifique de l'Algérie pendant les Années 1840, 1841, 1842, Publiée par Ordre du Gouvernement et avec le Concours d'une Commission Académique. Sciences Physiques. Zoologie III. Imprimerie Nationale, Paris, 590 pp.

Marsham T. 1802: Entomologia Britannica, sistens insecta Britanniae indigena, secundum methodum Linneanum disposita. I. Coleoptera. J. White, London, xxxi $+548 \mathrm{pp}$.

MaULIK S. 1926: The Fauna of British India, including Ceylon and Burma. Coleoptera. Chrysomelidae (Chrysomelinae and Halticinae). Taylor and Francis, London, xiv $+443 \mathrm{pp}$.

MauliK S. 1929: On the structure of the hind femur in halticine beetles. Proc. R. Entomol. Soc. Lond. 1929: 305-308.

MAULIK S. 1931: Coleoptera, Chrysomelidae, Eumolpinae, Galerucinae and Halticinae. Percy Sladen Trust Expedition, 1905. Trans. Linn. Soc. London (Ser. 2, Zool.) 19: 241-260.

Medvedev L. 1995: New species of Alticinae (Coleoptera, Chrysomelidae) from South Asia. Entomol. Basilien. 18: 479-488.
Medvedev L. 1996: The Chrysomelidae of Arabia. Fauna of Saudi Arabia 15: 215-263.

MonRós F. \& BechynÉ J. 1956: Über einige verkannte Chrysomeliden-Namen. Entomol. Arb. Mus. G. Frey 7: $1118-1137$.

Motschulsky V. 1860: Coleoptères de la Sibérie orientale et en particulier des rives de l'Amour. In Schrenck L. von (ed.): Reisen und Forschungen im Amurlande, vol. 2. St. Petersburg, pp. 77-257.

Mponda O.K.K., Gibbon D., Arthur E. \& Morse S. 1997: Involving farmers in the design of a low-input control programme for sesame flea beetles in Southern Tanzania. Exp. Agric. 33: 313-320.

NADEIN K.S. 2007: On the taxonomy and classification of the genus Psylliodes Latreille, 1825 (Coleoptera, Chrysomelidae, Galerucinae). Entomol. Basilien. Coll. Frey 29: 307-332.

OLIVIER G.A. 1789: Encyclopédie Méthodique, ou par Ordre de Matières; par une Societé de Gens de Lettres, de Savans et d'Artistes; Précedée d'un Vocabulire Universel, Servant de Table pour tout l'Ouvrage, Ornée des Portraits de MM. Diderot \& D'Alembert, Premiers Editeurs de l'Encyclopédie. Histoire Naturelle. Insectes. Tome IV. Panckoucke, Paris, 519 pp.

OLIVIER G.A. 1808: Entomologie, ou Histoire Naturelle des Insectes, avec leurs Caractères Génériques et Spécifiques, leur Description, leur Synonymie et leur Figure Enluminée. Coléoptères, Tome VI. Badouin, Paris, 501 pp.

Palisot de Beauvois A.M.F.J. 1812: Essai d'une Nouvelle Agrostographie, ou, Nouveaux Genres des Graminées: Avec Figures Représentant les Caractères de tous les Genres. Imprimé de l'auteur, Paris, xxiv + 182 pp., xxv pls.

Petitpierre E. 2000: Fauna Iberica, 13. Coleoptera Chrysomelidae 1. Museo National de Ciencias Naturales, Consejo Superior de Investigaciones Cientificas, Madrid, $521 \mathrm{pp}$.

PIC M. 1921: Notes diverses, descriptions et diagnoses. Echange 37: 1-6, 9-10, 13-15.

Pollard D.G. 1957: Halticinae of the Sudan. Bull. Entomol. Res. 47: 73-87.

Santiago-Blay J.A. 2004: Leaf-mining chrysomelids. In Jolivet P., Santiago-Blay J.A. \& Schmitt M. (eds): New Developments on the Biology of Chrysomelidae. SPB Academic Publishing, The Hague, pp. 305-306 and pp. 1-83 of the enclosed CD-ROM.

SAVINI V. \& FURTh D.G. 2001: The status of Heikertingerella, Monotalla, Pseudodibolia, and Sphaeroderma (Coleoptera: Chrysomelidae: Alticinae) in the New World. Proc. Entomol. Soc. Wash. 103: 903-912.

SAVINI V. \& FurTh D.G. 2009: Bubiscus, un género nuevo neotropical (Coleoptera, Chrysomelidae, Alticinae). Bol. Soc. Entomol. Aragon. 44: 53-61.

Sharp D. 1886: On New Zealand Coleoptera, with description of new genera and species. Trans. R. Dublin Soc. 3: 2351-2456.

Scherer G. 1959: Die Alticiden-Ausbeute der Expedition des Museums G. Frey nach Nigeria-Kamerun 1955/56 (Col. Phytoph.). Entomol. Arb. Mus. G. Frey 10: 177-265.

SCHERER G. 1961: Bestimmungsschlussel der Alticinen-Genera Afrikas. Entomol. Arb. Mus. G. Frey 12: 251-288.

Scherer G. 1962a: Beitrag zur Kenntnis der Alticiden-fauna Zentral-Afrikas (Coleoptera: Chrysomelidae: Alticinae). Ann. Mus. R. Afr. Centr. (Ser. 8, Zool.) 113: 7-82.

Scherer G. 1962b: Alticinae (Coleoptera, Phytophaga) Fam. Chrysomelidae. Exploration du Parc National de la Garamba. Mission H. De Saeger (1949-1952). Institut Parcs Nationaux du Congo et du Rwanda (Bruxelles) 31: 3-86. 
Scherer G. 1963: Beitrag zur Kenntnis der Alticidenfauna Afrikas (Coleoptera, Chrysomelidae, Alticinae). Entomol. Arb. Mus. G. Frey 14: 648-684.

SCHERER G. 1969: Contribution à la connaissance de la faune entomologique de la Côte-d'Ivoire (J. Decelle, 1961-1964). XLIII. Coleoptera Chrysomelidae Alticinae. Ann. Mus. R. Afr. Centr. (Ser. 8, Zool.) 175: 365-371.

SCHERER G. 1970: Beitrag zur Kenntnis der Alticinae Afrikas (Coleoptera - Chrysomelidae - Alticinae). Entomol. Arb. Mus. G. Frey 21: 298-304.

SCHERER G. 1972: Coleoptera aus Nordostafrica. Chrysomelidae: Alticinae. Notul. Entomol. 52: 1-17.

SCHERER G. 1978: Missione 1965 del professor Giuseppe Scortecci nello Yemen (Arabia meridionale). Coleoptera Chrysomelidae: Alticinae. Atti Soc. Ital. Sci. Nat. Mus. Civ. Stor. Nat. Milano 119: 264-266.

SCHERER G. 1983: Review of the genus Chirodica Germar. Entomol. Arb. Mus. G. Frey 31-32: 173-175.

SCHERER G. 1985: Yemenaltica, eine neue Gattung aus dem Yemen (Coleoptera Chrysomelidae Alticinae). Atti Soc. Ital. Sci. Nat. Mus. Civ. Stor. Nat. Milano 126: 85-88.

SCHERER G. 1987: The Genus Torodera Weise (Coleoptera Chrysomelidae - Alticinae). Entomol. Arb. Mus. G. Frey 35-36: 67-71.

SCHERER G. \& Boppré M. 1997: Attraction of Gabonia and Nzerekorena to pyrrolizidine alkaloids - with description of 13 new species and notes on male structural peculiarities (Insecta, Coleoptera, Chrysomelidae, Alticinae). Spixiana 20: 7-38.

SClater P. 1858: On the general distribution of the members of the Class Aves. J. Linn. Soc. Lond. (Zool.) 2: 130-145.

Seeno T.N. \& Wilcox J.A. 1982: Leaf beetle genera (Coleoptera, Chrysomelidae). Entomography 1: 1-221.

Selman B.J. 1963: Coléopterès Chrysomelides récoltés par M.J. Mateu dans l'Ennedi et au Tchad. Bull. Inst. Fr. Afr. Noire (A) 25: 1148-1162.

Selman B.J. 1968: A revision of the genus Eriotica Harold (Halticidae: Coleoptera). J. Nat. Hist. 2: 247-251.

Stephens J.F. 1829: The Nomenclature of British Insects. A Compendious List of such Species as are Contained in the Systematic Catalogue of British Insects, and of those Discovered Subsequently to its Publication; Forming a Guide to their Classification. Baldwin \& Craddock, London, $68 \mathrm{pp}$.

STEPHENS J.F. 1831: Illustrations of British Entomology; or, a Synopsis of Indigenous Insects: Containing their Generic and Specific Distinctions; with an Account of their Metamorphoses, Times of Appearance, Localities, Food, and Economy, as far as Practicable. Mandibulata. Vol. IV. Baldwin \& Craddock, London, $366 \mathrm{pp}$.

Thomson J. 1858: Voyage au Gabon. Arch. Entomol. 2: 9-471.

WeISE J. 1886: In Erichson W.F. (ed.): Naturgeschichte der Insecten Deutschlands. Erste Abtheilung. Coleoptera. Sechster Band, Vierte Lieferung, Bogen 37-48. Nicolaische Verlags-Buchhandlung, Berlin, pp. 569-768.

WeISE J. 1887: Neuer sibirische Chrysomeliden und Coccinelliden nebst Bemerkungen über früher beschriebene Arten. Arch. Naturgesch. 53: 164-209.

WeISE J. 1888: In Erichson W.F. (ed.): Naturgeschichte der Insecten Deutschlands. Erste Abtheilung. Coleoptera. Sechster Band, Fünfter Lieferung, Bogen 49-60. Nicolaische Verlags Buchhandlung, Berlin, pp. 769-960.
WeIse J. 1889: Insecta a Cl. G.N. Potanin in China et in Mongolia novissime Lecta. IX. Chrysomelidae et Coccinellidae. Horae Soc. Entomol. Ross. 23: 560-653.

WeISE J. 1895: Neue Chrysomeliden nebst synonymischen Bemerkungen. Dt. Entomol. Z. 1895: 327-352.

WeISE J. 1901: Einige neue afrikanische Chrysomeliden. Dt. Entomol. Z. 2: 301-310.

WeIse J. 1902a: Afrikanische Chrysomeliden. Arch. Naturgesch. 68: 119-174.

WeISE J. 1902b: Afrikanische Halticinen. Dt. Entomol. Z. 1902: 301-304.

WeISE J. 1903: Afrikanische Chrysomeliden. Arch. Naturgesch. 69: 197-226.

WeISE J. 1907a: Chrysomelidae et Coccinellidae aus dem Spanischen Guinea. Mem. R. Soc. Esp. Hist. Nat. 1: 379-403.

WeISE J. 1907b: Neue Chrysomeliden und Coccinelliden von der Ausbeute der Herren Oskar Neumann und Baron von Erlanger in Abyssinien. Arch. Naturgesch. 73: 210-232.

WeISE J. 1910a: Chrysomelidae und Coccinellidae. In Sjostedt Y. (ed.): Ergebnisse der schwedischen Expedition nach Kilimandjaro. Vol. 7. P. Palmquists Aktiebolag, Stockholm, pp. 153-266.

WEISE J. 1910b: Chrysomelidae von Madagaskar, den Comoren und den Inseln Ostafrikas. In Voeltzkow A. (ed.): Reise in Ostafrika in den Jahren 1903-1905, Band II, Heft 5. E. Schweizerbartsche Verlagsbuchhandlungen, Nägele \& Dr. Sproesser, Stuttgart, pp. 419-506.

WeISE J. 1911: Neue Chrysomeliden. Ann. Soc. Entomol. Belg. 55: $166-175$.

WeISE J. 1912: Chrysomelidae. In Schubotz H. (ed.): Wissenschaftiche Ergebnisse der Deutschen Zentral-AfrikaExpedition 1907-1908 unter Führung Adolf Friefrichs, Herzogs zu Mecklenburg. Klinkahrdt \& Biermannn, Leipzig, 127-163 pp.

WeISE J. 1915: Chrysomelidae and Coccinellidae: In Schubotz H. (ed.): Ergebnisse der Zweiten Deutschen Zentral-AfrikaExpedition 1910-1911 unter Führung Adolf Friefrichs, Herzogs zu Mecklenburg. Klinkahrdt \& Biermannn, Leipzig, pp. $155-184$.

Weise J. 1916: Synonymische Mitteilungen. Dt. Entomol. Z. 1916: $37-41$.

WeISE J. 1919: Afrikanische Chrysomeliden und Coccinelliden. Arch. Naturgesch. 83: 174-207.

WeISE J. 1922: Chrysomeliden der Indo-Malayischen Region. Tijdschr. Entomol. 65: 39-130.

WeISE J. 1923: Results of Dr. E. Mjöberg's Swedish Scientific Expeditions to Australia 1910-1913. 31. Chrysomeliden und Coccinelliden aus Queensland. Arkiv Zool. 15: 1-150.

WeISE J. 1924: Zoological results of the Swedish expedition to Central Africa, 1921. Insecta 7. Chrysomelidae und Coccinellidae. Arkiv Zool. 16: 1-30.

WeISE J. 1926: Über Bekannte und neue Chrysomeliden und Coccinelliden aus dem Reichmuseum zu Stockholm. Arkiv Zool. 18: 13-34.

Westwood J.O. 1840: An Introduction to the Modern Classification of Insects, Vol. 2. Longman, Orme, Brown, Green, and Longmans, London, $587 \mathrm{pp}$.

Westwood J.O. 1862: Description and figures of a new genus and species of Gallerucidae. J. Entomol. 1: 216-218.

Wollaston T.V. 1867: Coleoptera Hesperidium, being an Enumeration of the Coleopterous Insects of the Cape Verde Archipelago. Van Voorst, London, 285 pp.

Received December 29, 2009; revised and accepted March 2, 2010 